MICROWAVE-AIDED SYNTHESIS AND APPLICATIONS OF GOLD AND NICKEL NANOPOROUS METAL FOAMS

A Thesis
presented to
the Faculty of the Graduate School
at the University of Missouri-Columbia
In Partial Fulfillment
of the Requirements for the Degree
Master of Science
ZHIFENG LU

Dr. Sheila N. Baker, Thesis Supervisor

DEC 2013 
The undersigned, appointed by the dean of the Graduate School, have examined the thesis entitled

\section{MICROWAVE-AIDED SYNTHESIS AND APPLICATIONS OF GOLD AND NICKEL NANOPOROUS METAL FOAMS}

\section{Presented by Zhifeng Lu,}

a candidate for the degree of master of Science,

and hereby certify that, in their opinion, it is worthy of acceptance.

Professor Sheila N. Baker

Professor Paul C.H. Chan

Professor Hao Li 


\section{DEDICATION}

This thesis is dedicated to my great family and friends that have helped and supported me all the way since the beginning of my research. I could not have done it without you.

Also, this thesis is dedicated to all those who believe the richness of learning. 


\section{ACKNOWLEDGEMENTS}

First and foremost, I would like to express my sincere gratitude to Dr. Sheila N. Baker for the valuable guidance and advice. She inspired me greatly to work in my research. Her willingness to motivate me contributed tremendously to my research project in the past two years.

I would also like to thank Dr. Paul Chan and Dr. Hao Li for their willingness to be members of my thesis committee and for offering me with a good environment, facilities and kind help to complete this research project. Also, I wish to thank Sijia Hu who has been supporting me with helpful suggestions and assurance throughout the whole research project. In addition, I wish to thank all the members of Dr. Sheila N. Baker's research group for their help and encouragement. Finally, an honorable mention goes to my family and friends for their understandings and supports on us in completing this project. 


\section{TABLE OF CONTENTS}

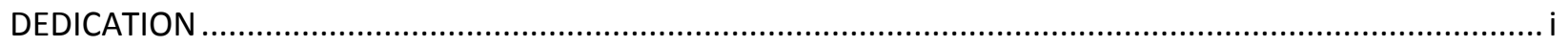

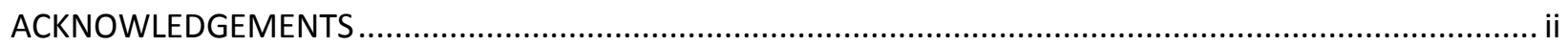

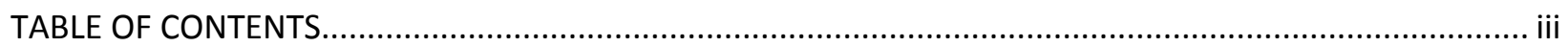

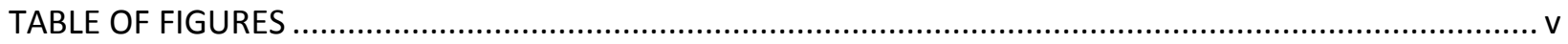

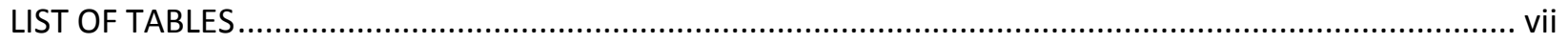

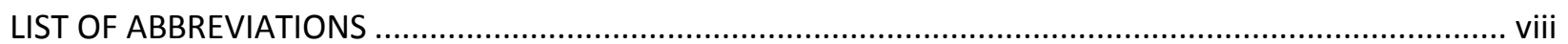

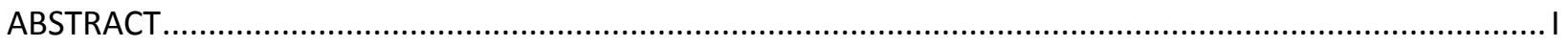



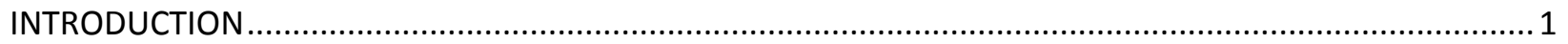

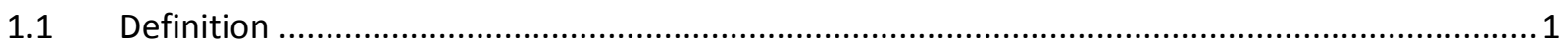

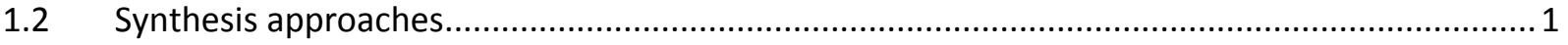

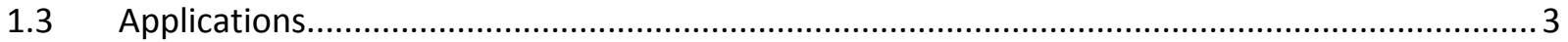

1.3.1. Surface enhanced Raman spectroscopy ........................................................................ 3

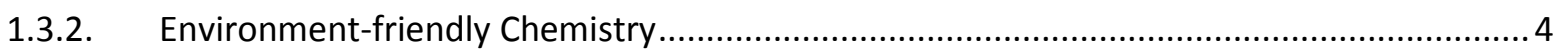

1.3.3. Energy storage and conversion ................................................................................... 5

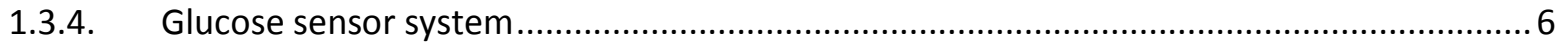

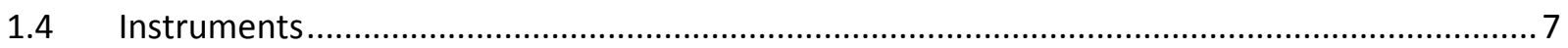

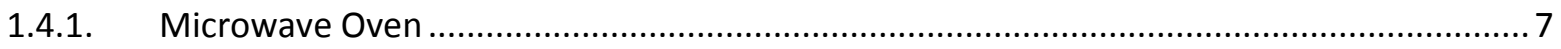

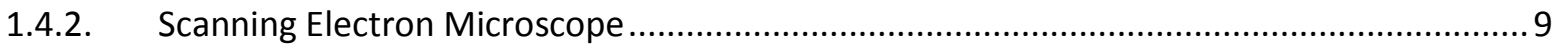

1.4.3. Surface-enhanced Raman Spectroscopy...................................................................... 10

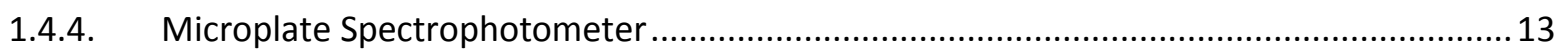

$1.5 \quad$ Motivation and Objective for Research ............................................................................. 14



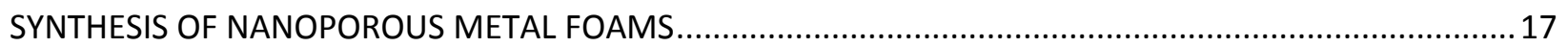

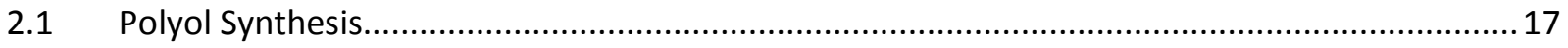



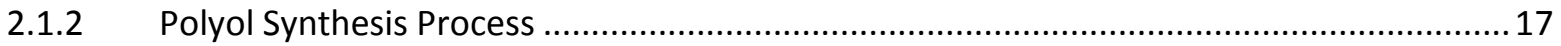

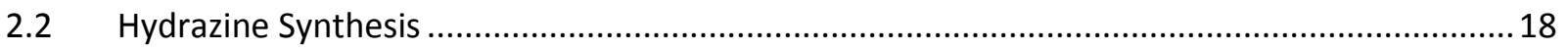

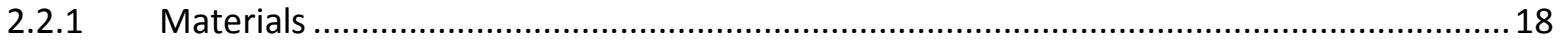

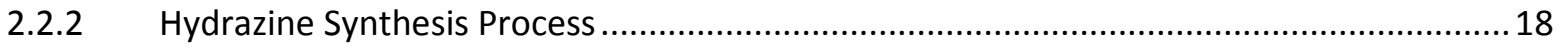




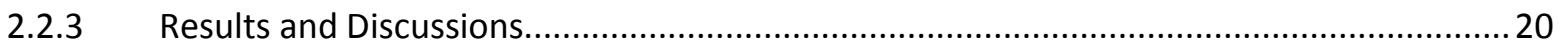

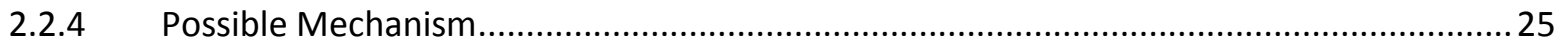

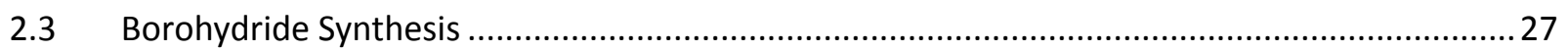

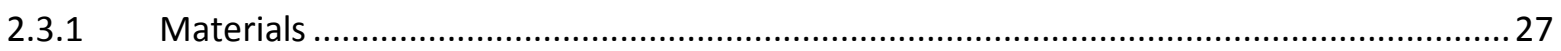

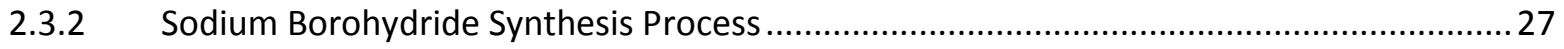

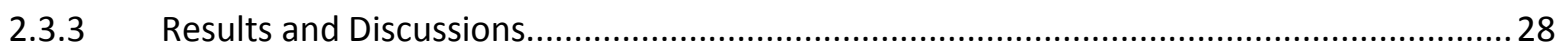

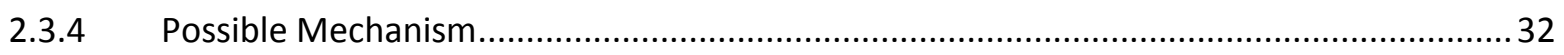

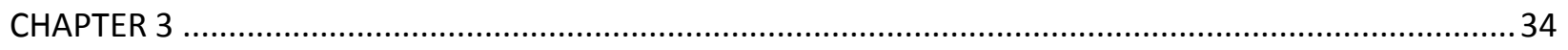

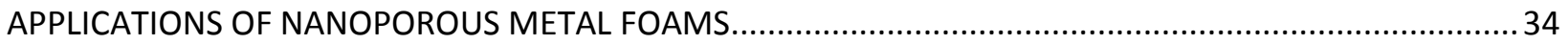

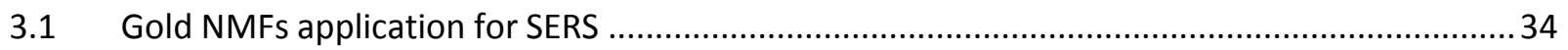

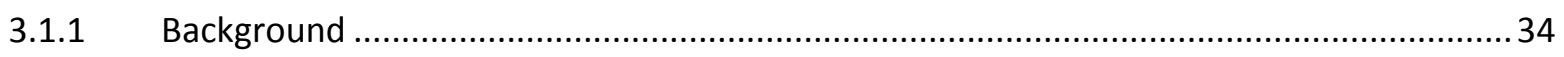

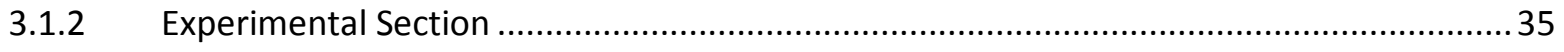

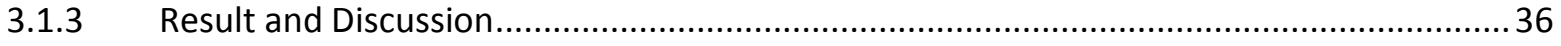

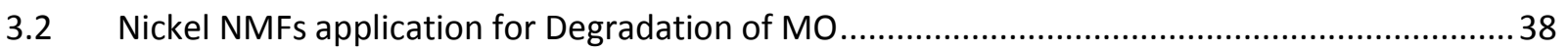

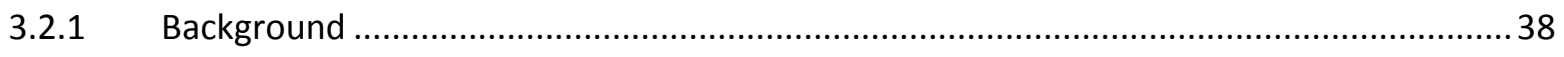

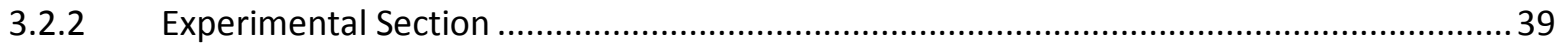

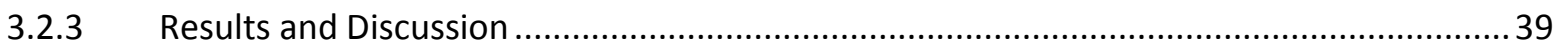

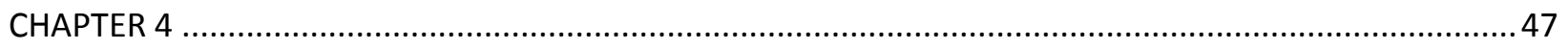



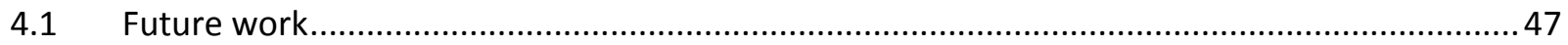

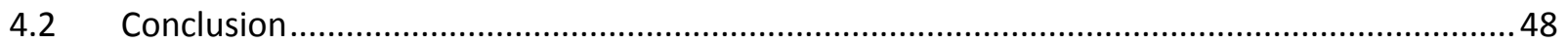

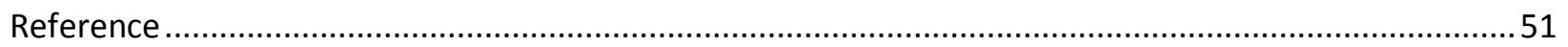




\section{TABLE OF FIGURES}

Figure 1-1 Image of CEM microwave used for the synthesis of all NMFs............................................. 8

Figure 1-2 Image of Hitachi S-4700 SEM. (University of Missouri-Columbia 1997)................................ 10

Figure 1-3 Energy level diagram of Raman scattering and Rayleigh scattering...................................... 11

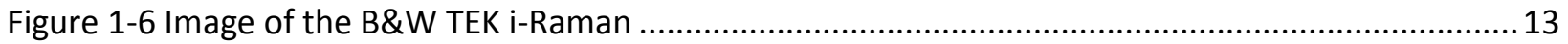

Figure 1-7 Image of BioTek Powerwave HT Microplate Spectrophotometer for the detection of degradation of MO solution immersed with nickel NMFs.

Figure 2-1 15K magnification SEM image of nanoporous gold foams reduced by hydrazine with EG

involved

Figure 2-2 30K magnification SEM image of nanoporous gold foams reduced by hydrazine with EG

involved.

Figure 2-3 10K magnification SEM image of nanoporous gold foams reduced by hydrazine without EG

involved

Figure 2-4 20K magnification SEM image of nanoporous gold foams reduced by hydrazine without EG

involved. 23

Figure 2-5 5K magnification SEM image of nanoporous nickel foams reduced by hydrazine...... .24

Figure 2-6 15K magnification SEM image of nanoporous nickel foams reduced by hydrazine. 25

Figure 2-7 40K magnification SEM image of nanoporous gold foams reduced by sodium borohydride with

EG involved .29

Figure 2-8 70K magnification SEM image of nanoporous gold foams reduced by sodium borohydride with EG involved 29

Figure 2-9 5K magnification SEM image of nanoporous nickel foams reduced by sodium borohydride... 30 Figure 2-10 50K magnification SEM image of nanoporous nickel foams reduced by sodium borohydride 
Fig 2-11 5K magnification SEM image of nanoporous gold foams reduced by sodium borohydride without EG

Figure 2-12 20K magnification SEM image of nanoporous gold foams reduced by sodium borohydride

without EG

Figure 3-1 Raman spectra of R6G molecules deposit on surface of gold NMFs: (a) $1.0 \times 10^{-5} \mathrm{M}$; (b) $2.1 \times$

$10^{-6} \mathrm{M}$; (c) $1.0 \times 10^{-6} \mathrm{M}$; (d) $5.2 \times 10^{-7} \mathrm{M}(\mathrm{e}) 1.0 \times 10^{-8} \mathrm{M}$. The spectra have been scaled and vertically

shifted to enhance the clarity of the presentation.

Figure 3-2 UV-Vis spectra of the MO solution $\left(2 \times 10^{-3} \mathrm{~mol} / \mathrm{L}\right)$ after different submersion time at room temperature.

Figure 3-3 UV-Vis spectra of the MO solution $\left(5 \times 10^{-4} \mathrm{~mol} / \mathrm{L}\right)$ after different submersion time at room

temperature.

Figure 3-4 UV-Vis spectra of the MO solution $\left(2 \times 10^{-5} \mathrm{~mol} / \mathrm{L}\right)$ after different submersion time at room

temperature.

Figure 3-5 Time variation of MO concentration after immersion of nickel NMFs, different concentration

of $\mathrm{MO}$ solution were also provided.

Figure 3-6 Time variation of MO concentration after the immersion of nickel NMFs with different

concentration of $\mathrm{MO}$ solution.

Figure 3-7 Time variation of MO concentration after the immersion of nickel NMFs with $2 \times 10^{-5} \mathrm{~mol} / \mathrm{L} \mathrm{MO}$

solution 


\section{LIST OF TABLES}

Table 1 Kinetics constants for increased MO concentration ................................................................ 46 


\section{LIST OF ABBREVIATIONS}

Ethylene Glycol $\quad$ EG

$\begin{array}{ll}\text { Methyl orange } & \text { MO }\end{array}$

Hydrazine $\quad \mathrm{N}_{2} \mathrm{H}_{4}$

$\begin{array}{ll}\text { Sodium Borohydride } & \mathrm{NaBH}_{4}\end{array}$

Sodium Chloride $\quad \mathrm{NaCl}$

$\begin{array}{ll}\text { Near-infrared radiation } & \text { NIR }\end{array}$

Nanoporous Metal Foams $\quad$ NMFs

Poly (vinyl pyrrolidone) $\quad$ PVP

$\begin{array}{ll}\text { Rhodamine 6G } & \text { R6G }\end{array}$

Scanning Electron Microscope $\quad$ SEM

Surface - enhanced Raman Spectroscopy $\quad$ SERS 


\section{ABSTRACT}

In the field of nanoscience, nanoporous metal foams are a representative type of nanostructured materials, representing the ultimate form factor of a metal. They possess the hybrid properties of metal and nanoarchitectures, including the following properties such as good electrical and thermal conductivity, catalytic activity and high surface area, ultralow density, high strength-to-weight ratio. The outstanding properties bring the nanoporous metal foams to a wide range of applications, especially in the field of sensor system, energy storage and chemical catalyst. A new method of synthesis developed recently is presented for nanoporous metal foams of gold and nickel. The goal of this study is for the synthesis process of NMFs of and some applications in research and realistic life.

Gold NMFs were produced by mixing gold chloride with ethylene glycol, ethanol, and reducing agent, and heating at $150{ }^{\circ} \mathrm{C}$ for 5 min with a CEM microwave. Both hydrazine and sodium borohydride were applied as the reducing agent for this redox reaction. Nickel NMFs were produced through the similar procedure with a little difference in the heating condition of $50 \mathrm{~W}$, instead of $150{ }^{\circ} \mathrm{C}$, with either hydrazine or sodium borohydride as the reducing agent.

Gold NMFs were applied in surface-enhanced Raman spectroscopy (SERS) as a substrate. It is presented that with the presence of gold NMFs, the detection of the rhodamine 6G (R6G), a model analyte, can be enhanced significantly. The limit of detection for rhodamine $6 \mathrm{G}$ was found to be $5.2 \times 10^{-7} \mathrm{M}$ in this research. Nickel NMFs 
was applied to degrade methyl orange (MO). An aqueous $\mathrm{MO}$ solution will turn nearly colorless after only $10 \mathrm{~h}$ of mixing with $0.025 \mathrm{~g}$ of nickel NMFs at room temperature under dark condition. In order to study the kinetics of the degradation reaction, $\mathrm{MO}$ solution with different initial concentration were used. This application of Ni NMFs is applicable as waste treatment of industrial water and to protect the environment. 


\section{CHAPTER 1}

\section{INTRODUCTION}

\subsection{Definition}

Nanoporous materials are a set of materials with large porosities (greater than 0.4 ), and pore diameters between 1-100 nm. (G Q Lu 2005) In the field of nanoscience, nanoporous metal foams are a representative type of nanostructured materials, representing the ultimate form factor of a metal. They possess the hybrid properties of metal and nanoarchitectures, including the following properties such as good electrical and thermal conductivity, catalytic activity and high surface area, ultralow density, high strength-to-weight ratio. The nanoporous metal foams are also distinguished over the bulk forms of metals in catalytic activity and plasmonic resonance due to the size-effect enhancements. (Tappan, Steiner et al. 2010)

\subsection{Synthesis approaches}

Compared with other nanoporous materials, nanoporous metal foams were developed late. The bottom-up approaches are not working well in the preparation of metal foams as they are in the preparation of non-metallic nanoporous foams. For example, aerogels are widely used in the synthesis but no synthetic pathways for gel with metallic backbonds have been demonstrated yet. Otherwise, the problem of the length-scale- 
dependent phenomena in scaling to nanometer dimensions need to be solved before the top-down approaches useful in preparing macrocellular metal foams can be applied to the synthesis of NMFs. (Tappan, Steiner et al. 2010)

There are some commonly used approaches to produce the NMFs presented so far, including the templating and dealloying approaches, sol-gel approaches, pyrolysis of metal salt or Dextran Pastes and combustion synthesis. (Tappan, Steiner et al. 2010)

The conventional synthesis methods are well suited for metal foams with large pores size from $200 \mu \mathrm{m}$ to $2 \mathrm{~mm}$. However, techniques such as selective dealloying are usually required for the synthesis of nanoporous metal foams with pore size less than 100nm. (Ni, Wu et al. 2012)

A preparative route of porous magnesia is reported by Han and Zhou group to fabricate pores in magnesia through in situ carbonization. Using P123 and PEO as templates and magnesium nitrate as precursor, magnesia foam materials are synthesized via one-pot pathway.(Han, Zhou et al. 2013) Another research group also presents a one-pot approach for the scalable synthesis of nanoporous $\mathrm{Ni}$ foams composed of nanowires under atmospheric pressure. As-prepared nanoporous $\mathrm{Ni}$ foams can be further transformed to different functional nanoporous foams.(Ni, Wu et al. 2012) A synthesis method that exploits a metal-organic frameworks (MOFs)-driven, self-templated, without using structure-directing surfactants route toward hierarchically nanoporous metal oxides via thermolysis under inert atmosphere is reported by Kim et al. (Kim, Lee et al. 2013) 
For the purpose of green chemistry, microwave assisted synthesis is introduced and attracts more and more interests. In the past, only when all the other options to drive a particular reaction are failed, or exceedingly long times or high temperatures are required for a reaction. Due to the availability in the lab and the advantages over the conventional heating, including the uniform heating, high efficiency of heating, reduction in unwanted side reaction and low operating cost, we choose to introduce the microwave irradiated approach into the synthesis process.(E.Karthikeyan 2011)

\subsection{Applications}

The nanoporous metal foams have three-dimensional structures comprised of interconnected metallic particles or filaments exhibiting a porosity of no less than $50 \%$ and in which sub-micro pores, including micropores, mesopore, and macropores 50 $1000 \mathrm{~nm}$ in diameter, measurably contribute to the specific surface area of the foam. NMFs straddle previously unoccupied parameter space in the plot of pore size versus relatively density for porous, low-density metallic materials. The outstanding properties, for example, low relative density ( $\left.\rho_{\text {foam }} / \rho_{\text {bulk}}\right)$, high specific surface area, enhanced plasmonic behavior, and size-effect-enhanced catalytic behavior bring the nanoporous metal foams to a wide range of applications, especially in the field of sensor system, energy storage and chemical catalyst. (Tappan, Steiner et al. 2010)

\subsubsection{Surface enhanced Raman spectroscopy}

Raman spectroscopy is an important analytical technique for chemical and biological analysis since the information on molecular structures, surface processes and interface 
reactions that can be extracted from experimental data is precious for the research study. The possibility of achieving low detection limit with normal Raman spectroscopy is at a low level due to the inherently weak Raman cross-section.(Vo-Dinh 1998)

However, there is a remarkable 14-order-of-magnitude signal enhancement that will occur during Raman scattering from molecules on metallic nanostructures which turns the normally weak inelastic-scattering effect into a single-molecule spectroscopic probe. (Kneipp, Moskovits et al. 2007) The wide variety of methods using different types of solid and liquid SERS media increases the popularity of the SERS technique. Metal nanoparticle films and nanostructured substrates were developed and used as SERSactivate media for a wide variety of areas, following the development of roughened metal electrodes and metal colloids, in applications requiring reproducible results.(VoDinh 1998) Nanoporous gold with excellent thermal stability and chemical inactivity has been exploited as an attractive substrate for SERS applications because of its large surface area and bicontinuous porous structure in three dimensions. It has been reported that Nanoporous gold with the smaller pore size has stronger SERS enhancements.(Qian, Yan et al. 2007)

\subsubsection{Environment-friendly Chemistry}

With the development of textile, paper, plastics, leather, food and cosmetic industry in the recent decades, great amount of the synthetic organic dyes and pigments are used. However, most of the synthetic organic dyes are not bio-degradable and hence come out to threaten the environment, especially the aquatic environment, severely. The azo dyes are the kind of dyes widely applied in textile industries for their ease of synthesis, versatility and cost-effectiveness. While, the azo dyes with toxic, stable and soluble 
behavior are proposed to be degraded by the methods such as adsorption, filtration, sedimentation and photocatalytic action. Among various methods for organic dyes and chemicals, catalytic degradation has been demonstrated to be one of the most important, innovative and green technologies for water treatment. Due to the large surface-to-volume ratios and subsequent increase in reaction rate in catalysis, nanoporous metal foams, including gold, nickel and palladium, exhibit the ability of catalytic degradation for methyl orange (MO) which the bulk metals don't have. (Hakamada, Hirashima et al. 2012; Kumar, Kumar et al. 2013)

\subsubsection{Energy storage and conversion}

The design and fabrication of three-dimensional multifunctional architectures from the appropriate nanoscale building blocks, including the strategic use of void space and deliberate disorder as design components, permits a device to be re-examinable for producing or storing energy. The prime advantages one expects with the proposed 3D architectures for energy storage in batteries, in addition to the small areal footprint, are the short transport lengths for ions in the solid-state electrode as well as between the anode and cathode. The 3D design minimizes both distances yields concomitant improvements in power density. (Gates 2013)

Areal power capacity is reported to be significantly increased since the electrode geometries are extended from 2D to 3D. Nanoporous metal foams and the derivatives show the probability to be used to produce enhanced electrodes for batteries, such as high-surface-area porous zinc for zinc-air batteries. Additionally, nanoporous metal foams provide the thin struts and open porous "highways" to be an ideal environment for 
rapid mass transport of ions into and out of electrodes and in turn, faster discharging and recharging batteries.(Tappan, Steiner et al. 2010)

\subsubsection{Glucose sensor system}

The first use of commercially available three-dimensional porous $\mathrm{Ni}$ foam as a novel electro-chemical sensing platform for nonenzymatic glucose detection is reported by the research group leading with Wenbo Lu recently. In their studies, the porous $\mathrm{Ni}$ foams not only act as a working electrode, but also function as an effective electrocatalyst for electrooxidation of glucose. Compared to other detection methods, the electrochemical technique is a promising tool for constructing simple and low-cost sensors due to its remarkable features such as high sensitivity, simple instrumentation, reliability, selectivity, and ease of operation. Precious metals and their alloys were found to exhibit good catalytical activity toward electrooxidation of glucose and have been widely used for nonenzymatic glucose detection. However, precious metal-based catalysts suffer from high cost, limiting their practical applications. Researchers demonstrated that metal and metal oxides can be used as effective electrocatalysts in order to develop the low-cost, non-noble electrocatalysts. The Ni nanoparticles exhibit great enhancement in the electro-oxidation of glucose compared to other metallic nanoparticle-based electrodes. However, all the methods suffer from complex and time-consuming synthesis routes or the involvement of hazardous reagents. Accordingly, a $3 \mathrm{D} \mathrm{Ni}$ structure is an ideal electrode architecture for nonenzymatic glucose detection due to its interpenetrating network of electron and ion pathways for efficient ion and electron transport.(Lu, Qin et al. 2013) 


\subsection{Instruments}

\subsubsection{Microwave Oven}

Microwave heating is a dipolar phenomenon with frequencies that range from 0.3 to 300 $\mathrm{GHz}$, corresponding to wavelengths of $1 \mathrm{~cm}$ to $1 \mathrm{~m}$. The two main principles involved in microwave chemistry are the dipolar mechanism and the electrical conductor mechanism. The first mechanism occurs when the polar molecules attempt to follow a high frequency electric field and release enough heat to drive the reaction forward. The second mechanism occurs when the charge carriers, such as electrons and ions, are moved through the material under the influence of the electric field, resulting in a polarization. The induced currents and the electric residence of the conductor will cause heating in the irradiated sample.(Nadagouda, Speth et al. 2011)

The main advantages that distinguished the microwave heating over the conventional heating include the uniform heating throughout the material, high heating speed and efficiency, reduction in unwanted side reaction and side products and relatively low operating cost.(E.Karthikeyan 2011) 


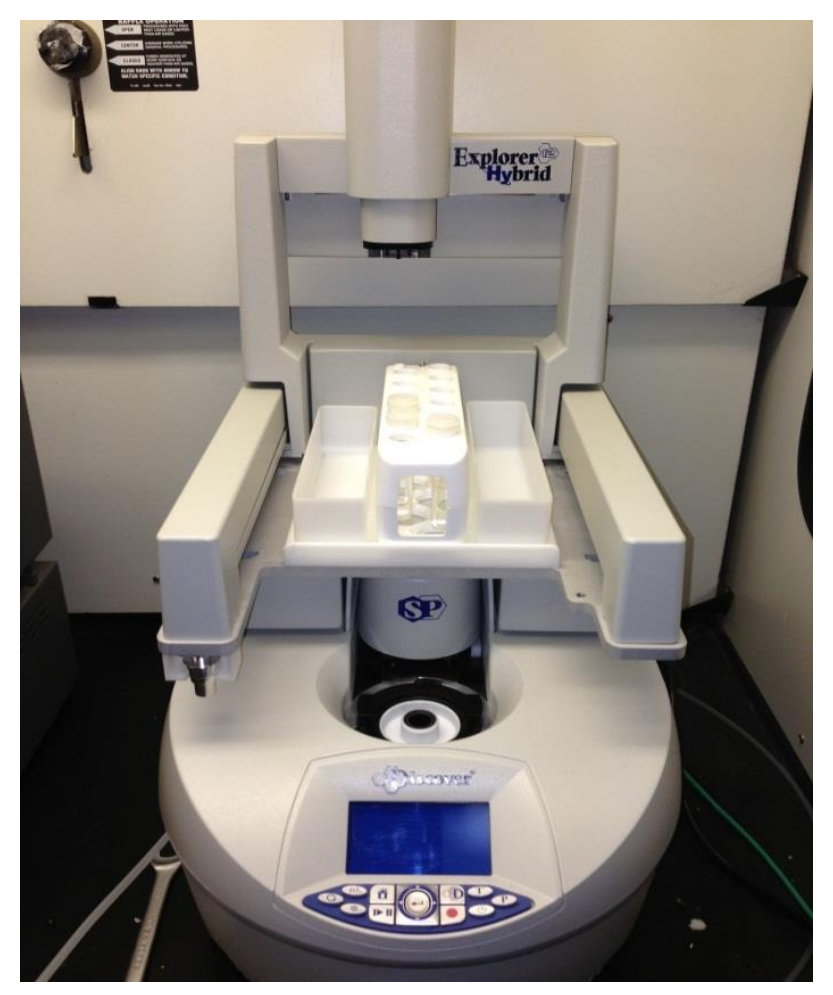

Figure 1-1 Image of CEM microwave used for the synthesis of all NMFs

For example, the silver nanoparticles have been produced mainly by chemical solution processes with aggressive chemical reducing agents, capping agents and organic solvents in the past. All kinds of the chemical agents can threaten the environment severely. Otherwise, Yugang Sun and co-workers report that at least 30 minutes are necessary for the shape-controlled synthesis of gold and silver nanoparticles. However, another research group also reported when applying the microwave irradiation, the irradiation heating process proceeds within 10s. All these excellent properties and the growing availability in laboratories promise the microwave irradiation to be a beneficial technology for research work. (Sun 2002; Hu, Wang et al. 2008) 


\subsubsection{Scanning Electron Microscope}

Early invented and improved in the 1930's, the scanning electron microscope (SEM) has been used as a powerful and professional technology for the examination of the surface structure of materials. SEM provides information on surface topography, crystalline structure, chemical composition and electrical behavior of the $1 \mu \mathrm{m}$ or so of specimen. It is widely used in the laboratory and in the research for its advantages over the optical microscopy, including higher magnifications (up to $1,000,000 x$ ) compared with $1000 x$ with optical and more information other than the surface topography.

The SEM equipment we use for the research is Hitachi S-4700 which is consisted of a microscope column, a specimen chamber, a vacuum system, a monitor, computer software and other controlling instruments. A Hitachi S-4700 uses a cold field emission because heat is not used to lower the work potential. An accelerating voltage ranging from 0.5 to $30 \mathrm{kV}$ promise a resolution of $1.5 \mathrm{~nm}$. Compared with what can be obtained with the optical microscopy, both the magnification (30x to 500,000x) and the depth of field can be many times greater.(Smith and Oatley 1955; Vernon-Parry 2000; Hafner 2007) 


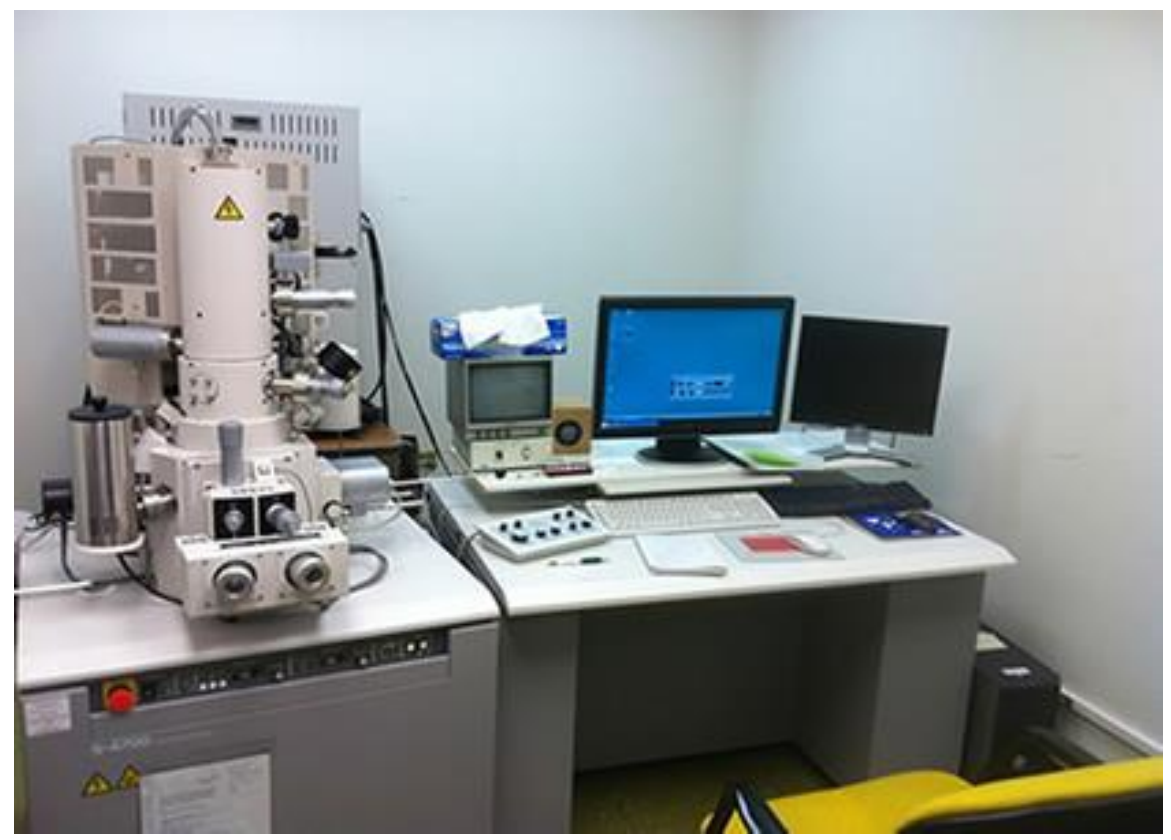

Figure 1-2 Image of Hitachi S-4700 SEM. (University of Missouri-Columbia 1997)

The Hitachi S-4700 utilizes a cold cathode field emitter composed of a single crystal of tungsten etched to a fine point. In cold field emission electron microscopy, an electric field to the tip instead of huge amount heat energy is applied to pull the electrons from the emitter. Two anodes are also applied for the acceleration of electrons. The high resolution of instrument is attributed to the smallest available beams emitted and the microscopic size of the electron source. (Alyamani and Lemine ; Hafner 2007)

\subsubsection{Surface-enhanced Raman Spectroscopy}

When photons are scattered from an atom or molecule, most photons are elastically scattered so that the scattered photons and the incident photons have the same energy, which is called Rayleigh scattering. In some other cases, when light interacts with matter, it can scatter inelastically from vibrational quantum states. A molecular-energy diagram can illustrate the inelastic scattering of photons from matter. The Ramanscattering (RS) signal appears shifted to lower energy than the excitation energy when 
the incident photons interact with a molecule in its vibrational ground, which is the Stokes Raman scattering. While if the incident photons interact with a molecule in its first-excited state, then the Raman-scattering signal will shift to higher energy, which is the Anti-Stokes Raman scattering. (Kneipp, Moskovits et al. 2007)



Figure 1-3 Energy level diagram of Raman scattering and Rayleigh scattering

The Raman Spectroscopy is a spectroscopic technique using the inelastic scattering of monochromatic light to observe vibrational, rotational, and other low-frequency modes in a system. As a result, it can be applied for the microscopic examination in a wide field including mineral, materials such as polymers and ceramics, cells, proteins and forensic trace evidence since water doesn't generally interfere with Raman spectral analysis.

The technique of Raman spectroscopy presents several advantages for microscopic analysis. For example, fixed or sectioned specimens are no longer required. Little amount of specimen is adequate for Raman spectroscopy to identify the species in the volume. Besides, time in the scale of seconds is enough for acquiring Raman spectra. Remote analysis can also be achieved with long optical fibers by what laser light and Raman scattered light can be transmitted. Another advantage of the Raman 
spectroscopy is the high resolution. However, there are still some disadvantages against the Raman spectroscopy. Due to the weak Raman Effect signals keep the detection sensitivity to an extremely low level and the instrumentation to be optimized at a high standard. Fluorescence is a common background issue since Raman signal would be overwritten by fluorescence emission signal.

Typically, a Raman system is consisted with four major components, including the excitation source (a laser), sample illumination system and light collection optics, wavelength selector, and detector such as photodiode array and CCD.

The Raman microscope applied in our research is i-Raman from B\&W Tek Inc. Figure 1-6 shows the image of entire instrument. This instrument is unique for its high resolution combined with field portability. Besides, the i-Raman spectrometer system applies a CleanLaze ${ }^{\circledR}$ technology with a line width $<0.3 \mathrm{~nm}$ when equipped with $785 \mathrm{~nm}$ and $830 \mathrm{~nm}$ laser to promise the correct center wavelength. In addition, the laser output power can be adjusted and controlled with the software from $0-100 \%$, allowing us to reduce the effect of the background noise and do the test within a relatively short time period. Light source with wavelengths of $532 \mathrm{~nm}, 785 \mathrm{~nm}$ and $830 \mathrm{~nm}$ are provided as options for the excitation that are ideal for demanding applications involving low concentrations and weak Raman scatters. Besides, Raman shift up to $4000 \mathrm{~cm}^{-1}$ could be reflected by the instrument and a TE cooled 2048 pixel CCD array can be achieved. 


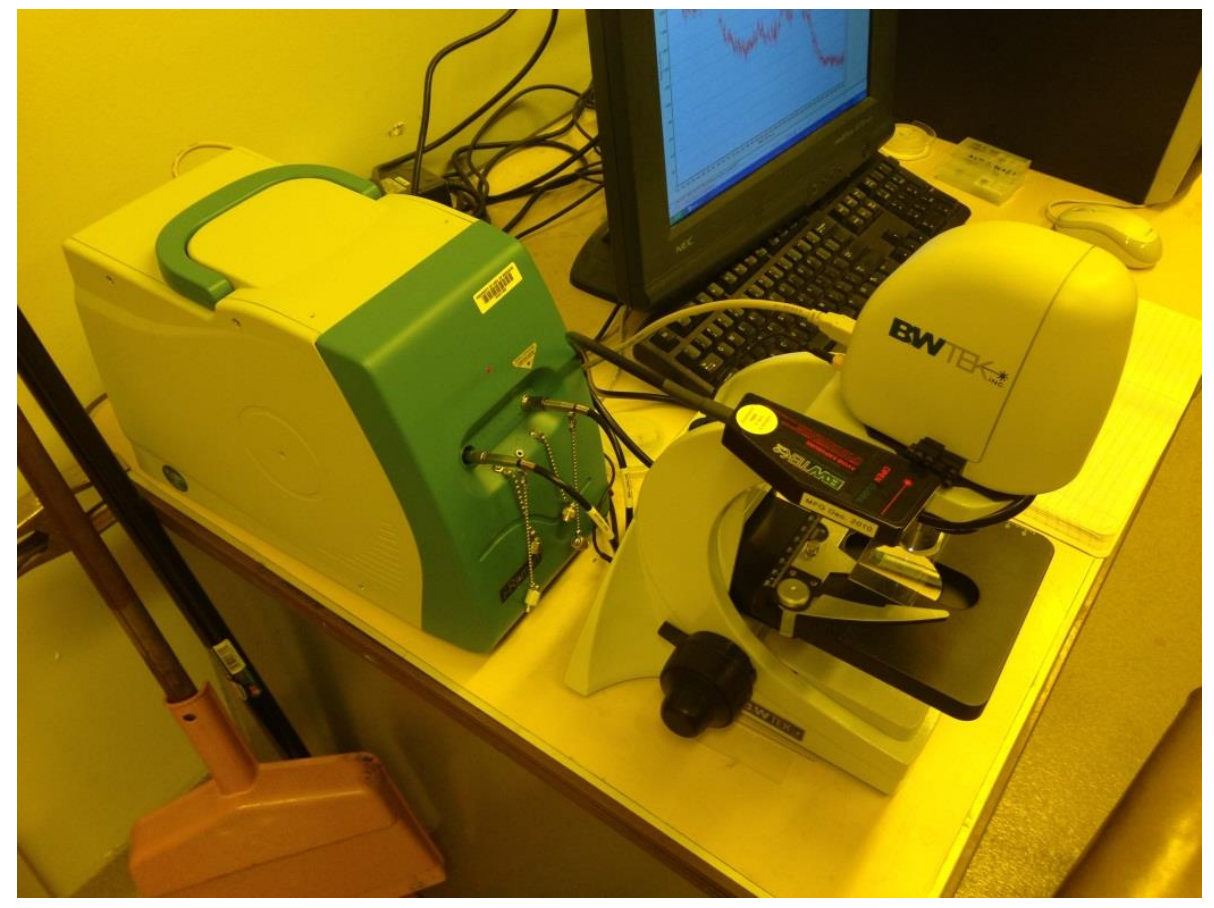

Figure 1-4 Image of the B\&W TEK i-Raman

\subsubsection{Microplate Spectrophotometer}

The plate reader, also called microplate spectrophotometer, was used in our research for the degradation processes of MO solution in the presence of nickel NMFs. In the research experiment, we used BioTek Powerwave HT Microplate Spectrophotometer, which is designed for a huge amount of applications requiring high speed, high accuracy and high reliability such as direct quantitation of nucleic acids using automated pathlength correction. This instrument is uniquely designed to provide the application flexibility due to its rugged hardware and proven optical performance. Gen5 Data Analysis software together with this plate reader offered a clear and operable system for the spectra reading and data collecting. 


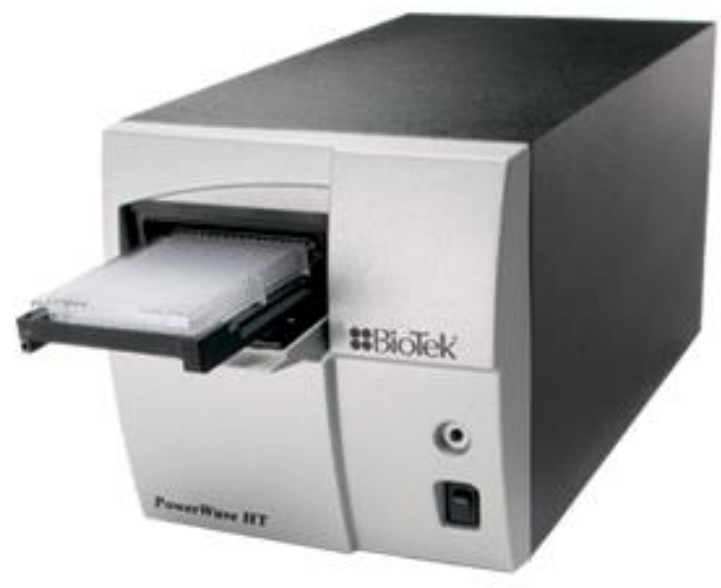

Figure 1-5 Image of BioTek Powerwave HT Microplate Spectrophotometer for the detection of degradation of MO solution immersed with nickel NMFs.

In addition, this instrument has many useful features, for example, no interference filter is required. The continuous wavelength selection from $200 \mathrm{~nm}$ to $999 \mathrm{~nm}$ with $1 \mathrm{~nm}$ increment for wavelength selection makes this instrument to meet almost all requirements. Additionally, it provides a fast reading. An entire $48-$ well plate can be read in 4 minutes. Moreover, the temperature of the test environment can be control as well.

\subsection{Motivation and Objective for Research}

The initial goal of this research was to develop a new synthesis route to produce gold and nickel nanoporous metal foams (NMFs) with microwave irradiation heating. A polyol synthesis using PVP, sodium chloride and ethylene glycol was firstly accepted referring to the paper from Benjamin Wiley et al. (Wiley, Herricks et al. 2004) However, this polyol process can only be applied to the synthesis of silver rather than gold or nickel. In order to achieve the goal, another synthesis is studied and developed. Referring to the 
report of the research group of Yue Wang et al, the reduction of hydrazine in glycerolethanol solution at different temperature can be used to produce the nanoporous structures of various kinds of metals. (Wang, Shi et al. 2012) Thus, we applied this route to our producing system. Another reductant, sodium borohydride, was found to replace the hydrazine to develop the product with different surface properties for my research.

Various methods on papers apply conventional heating instead of microwave. According to the research and mechanism of microwaves, many advantages are proved for the microwave irradiation process in study of nano-techniques. For instance, an efficient and uniform heating rate can be selectively directed towards a targeted area with microwave irradiation, which reduces the probability of side reactions and side products. As a result, the physicochemical properties and yield of the products are improved. These are the important facts that encourage the utilization of microwave in both manufacture industries and laboratory researches. This is also the main reason for us to apply microwave irradiation for our reaction. While there are still some challenges for the process of reactions, the most primary one is to control the concentration of the solution and the amount of the reductant, since the hydrazine and sodium borohydride are both strong reductants, especially the sodium borohydride, that can react with ethanol, the solvent of our solution. This is why we need magnetic stirring to obtain a uniform solution, further improving the chance of reactions and yield.

The next goal for my research was to apply the NMFs to the applications in industry and real life. Though huge amounts of useful applications were found for NMFs in a wide range of fields, some of them don't match the idea of green chemistry and can be only developed in research labs and experiments. Waste treatment instead of waste 
producing is the better way to apply the NMFs. For this purpose, we aim to utilize the NMFs into the treatment of azo dyes, which are widely used but toxic and quite stable in water. In our work, nanoporous nickel foams present the ability to be the efficient catalyst in degradation of methyl orange from dark orange to almost colorless.

Surface enhanced Raman spectroscope was developed for decades for the observation of signal from different analytes. Gold NMFs are engineered to perform as the substrate to mix with the analyst in order to enhance the signal. In our research, rhodamine $6 \mathrm{G}$ is used as the analyte to prove that a relatively low limit of detection can be achieved. In another word, the detecting ability can be improved with the gold NMFs as the substrate.(Kudelski 2005) 


\section{CHAPTER 2}

\section{SYNTHESIS OF NANOPOROUS METAL FOAMS}

\subsection{Polyol Synthesis}

\subsubsection{Materials}

All experiments were completed using Ultrapure Milliopore water (18.2 M $\Omega$ ). Silver nitrate (CAS\#7761-88-8, AgNO3, 299\%, MW: 169.87), ethylene glycol (CAS\#107-20-1, C2H6O2, $\geq 99 \%, \mathrm{MW}: 62.07$ ) and sodium chloride (CAS\#7647-14-5, $\mathrm{NaCl}, \geq 99 \%, \mathrm{MW}$ : 58.44) were purchased from Sigma Aldrich (St. Louis, MO). All materials were used without any further purification.

\subsubsection{Polyol Synthesis Process}

In the synthesis process, $3 \mathrm{ml}$ EG was first added into a microwave vial by $1000 \mu \mathrm{l}$ pipette. $0.94 \mathrm{~mol}$ silver nitrate, $0.125 \mathrm{~g} \mathrm{PVP}$ and $0.002 \mathrm{~g}$ sodium chloride was weighted out by a balance and added into the vial containing $3 \mathrm{ml} \mathrm{EG}$. A 20-minute-strring at room temperature is required, and then the vial with cap was dropped into the microwave oven for heating process. Sample foams were generated in the solution after heating at $150{ }^{\circ} \mathrm{C}$ for 5 minutes. Magnetic stirring was also applied through the entire irradiation heating process. The liquid was treated as the unwanted solution and poured into the hazardous unwanted bottles. The solid sample was moved into a $15 \mathrm{ml}$ plastic centrifuge tube by a spatula. Ethanol was then used for the sample washing. After ethanol was added to the tube with the sample in it, the tube was then centrifuged at 
$5000 \mathrm{rpm}$ for 4 minutes. The ethanol used for washing was removed to the unwanted bottles as well. With a spatula, the sample foams were taken out of the tube and put into a scintillation vial. A furnace was applied for the drying of the sample foams. Within 24 hours, we could obtain the completely dried sample foams. (Wiley, Herricks et al. 2004)

\subsection{Hydrazine Synthesis}

\subsubsection{Materials}

Gold (I) Chloride (CAS\#10294-29-8, AuCl, 99+\%, MW: 232.42), ethylene glycol


99.5\%, MW: 46.07) were purchased from ACROS Organics. Nickel (II) Chloride (CAS\#7718-54-9, NiCl2, 98\%, MW: 129.60), was obtained from ALDRICH. Hydrazine monohydrate (CAS\#7803-57-8, $\mathrm{H} 4 \mathrm{~N} 2 \cdot \mathrm{H} 2 \mathrm{O}, \geq 99 \%$, MW: 50.06) was purchased from Alfa Aesar Chemicals. All materials were used without any further purification.

\subsubsection{Hydrazine Synthesis Process}

$0.018 \mathrm{~g}$ gold chloride was first weight out and placed into a $10 \mathrm{ml}$ microwave vial with a microwave stir bar in it. The mixture of $1 \mathrm{ml}$ ethanol and $1 \mathrm{ml}$ of EG was added into the vial via pipette. The EG in the mixture was applied to prevent aggregation. After stirring for 5 minutes, the solution would be almost clear and $0.5 \mathrm{ml}$ hydrazine was added as the reducing agent. Another 15 minutes of stirring is necessary for success, which promise the components to be well dispersed. The vial was then capped and put into the microwave for heating. After the 5 minutes heating at temperature of $150 \stackrel{\circ}{ } \mathrm{C}$, nanoporous gold foams were generated. After the same washing process like polyol 
synthesis, the sample foams were centrifuged and dried. The sample foams were stored in a capped vial at last. From the calculation, $0.060 \mathrm{~g}$ gold chloride can produce $0.040 \mathrm{~g}$ nanoporous gold foams. The total weight of gold contained in $0.060 \mathrm{~g}$ gold chloride is $0.05085 \mathrm{~g}$. The yield of this synthesis process achieved $78.66 \mathrm{wt} \%$.

Another synthesis recipe was also developed in our lab because the product generated with the synthesis above encountered with some problems when applied to the application of Surface-Enhanced Raman Spectroscope, the EG used is a competitive organic agent preventing us to detect the analyte. As a result, that $1 \mathrm{ml} E G$ was replaced with another $1 \mathrm{ml}$ ethanol, in order to obtain the same concentration with the same amount of gold chloride. All the other steps were kept the same. After 5 minutes of microwave irradiation heating at $150{ }^{\circ} \mathrm{C}$, the nanoporous gold samples were produced. With this synthesis process, $0.047 \mathrm{~g}$ gold samples were generated with 0.060 g gold chloride, which means that the yield reached up to $92.42 \mathrm{wt} \%$, even better than the synthesis with ethylene glycol involved.

Another kind of metal foams, the nanoporous nickel foams, were also studied in our lab with this synthesis process. $0.014 \mathrm{~g}$ nickel chloride was weight and added into the mixture of $1 \mathrm{ml}$ of ethanol and $1 \mathrm{ml}$ of EG. The heating process was set the same as that for gold chloride, which is $150{ }^{\circ} \mathrm{C}$ for 5 minutes. But in the case of nickel, there would be a pressure problem occurs during the microwave irradiation heating process. The reaction released a great amount of gas which leaded the pressure increasing rapidly over the safety pressure of the microwave oven. For that reason, the microwave oven would stop automatically to protect the operator and the instrument. The heating period for producing nanoporous nickel foams decreased from 5 minutes as settled to 
around 2 minutes and 30 seconds. As a result, another process of heating was required then. The heating conditions were settled to be 50 watts for 2 minutes and a repeating process was involved to keep the heating time. After the nanoporous nickel foams were generated, the following steps including washing, centrifuging and drying were still required and operated as those for nanoporous gold foams. However, the yield of nanoporous nickel foams is much lower than gold foams. Only $0.020 \mathrm{~g}$ sample foams were produced from $0.085 \mathrm{~g}$ nickel chloride, which contains $0.0385 \mathrm{~g}$ nickel ions in total. The yield was $51.95 \mathrm{wt} \%$.

\subsubsection{Results and Discussions}

The SEM observation of our sample products was achieved by the Hitachi S-4700 SEM in the EMC of University of Missouri-Columbia. For the step of specimen preparation, the sample NMFs were placed on the specimen stub and then the stub can be placed on a lock screw, attached to the specimen exchange rod. The height could be adjusted by the specimen height gauge. In order to guarantee that all the ion pump readings and EAVC power switch were set correctly, the chamber needs to be vacuumed. Specimen exchange position was then checked to make sure all the axis control were in the right position and stage was not locked. After the preliminary operation, a prepared specimen was sent into the vacuum chamber using the specimen exchange rod.

After setting up the accelerating voltage and the current intensity of the electron gun was completed, the HV electron gun was switched on. The observation operation mode was set as ultrahigh resolution. Then the image could be read on the computer screen linked to the SEM. 
The following operation would be applied to obtain clear images we desired. The magnification was set to be low first to manually control the position of the specimen. When the region of interest of the specimen was located and focused correctly, a higher magnification would apply. The next step was to adjust the alignment. For beam align, just move the target into a circle beam. For aperture align, shifting in either $X$ or $Y$ direction of the image on the screen should be eliminated as possible as we can. The same procedure would be applied as well to the $\mathrm{X}$ and $\mathrm{Y}$ aligns. Then all the alignment adjustments were completed and relatively clear images would show on the screen.

The SEM images for the sample foams with the hydrazine synthesis are provided as followed. Figure 2-1 - 2-4 shows the microstructure of the gold NMFs with different magnifications and reductants.

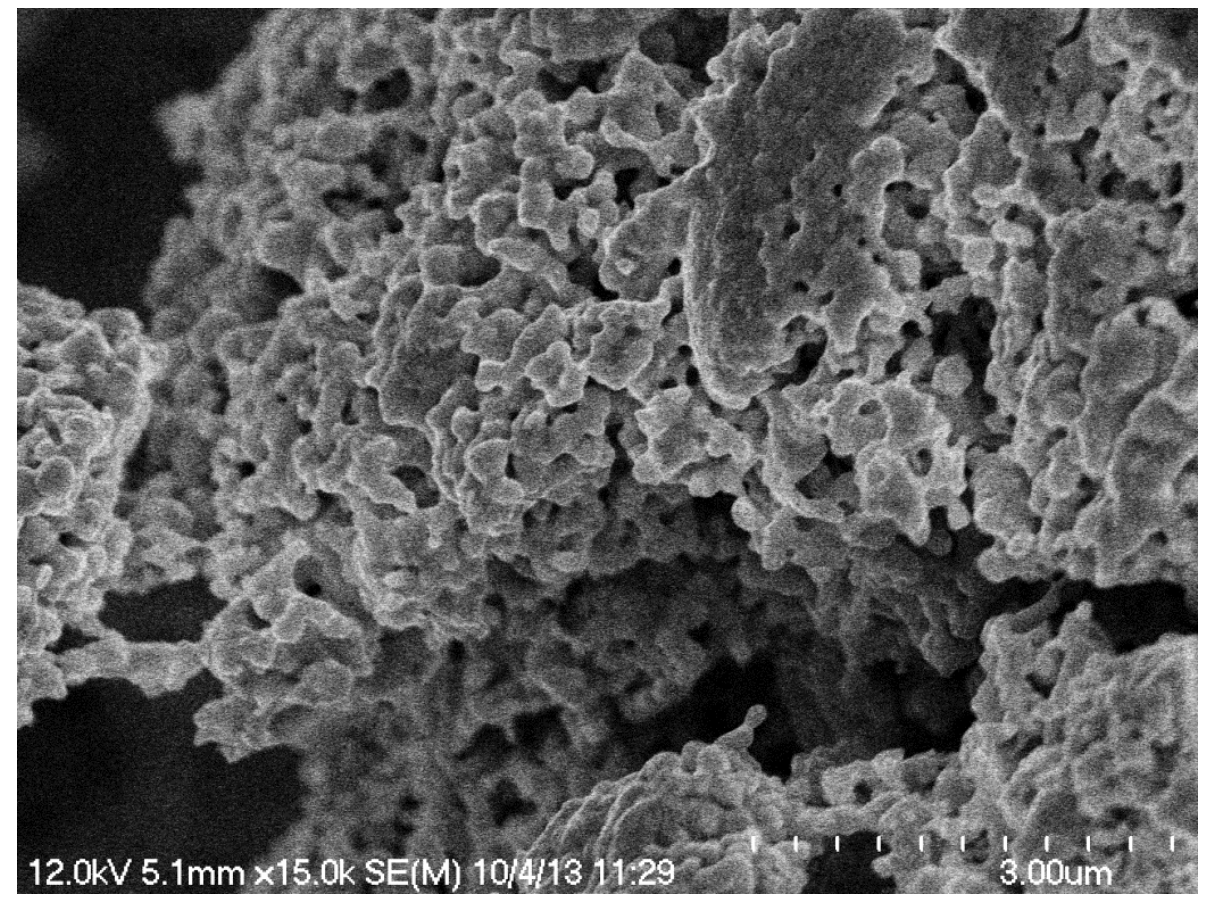

Figure 2-1 15K magnification SEM image of nanoporous gold foams reduced by hydrazine with EG involved 


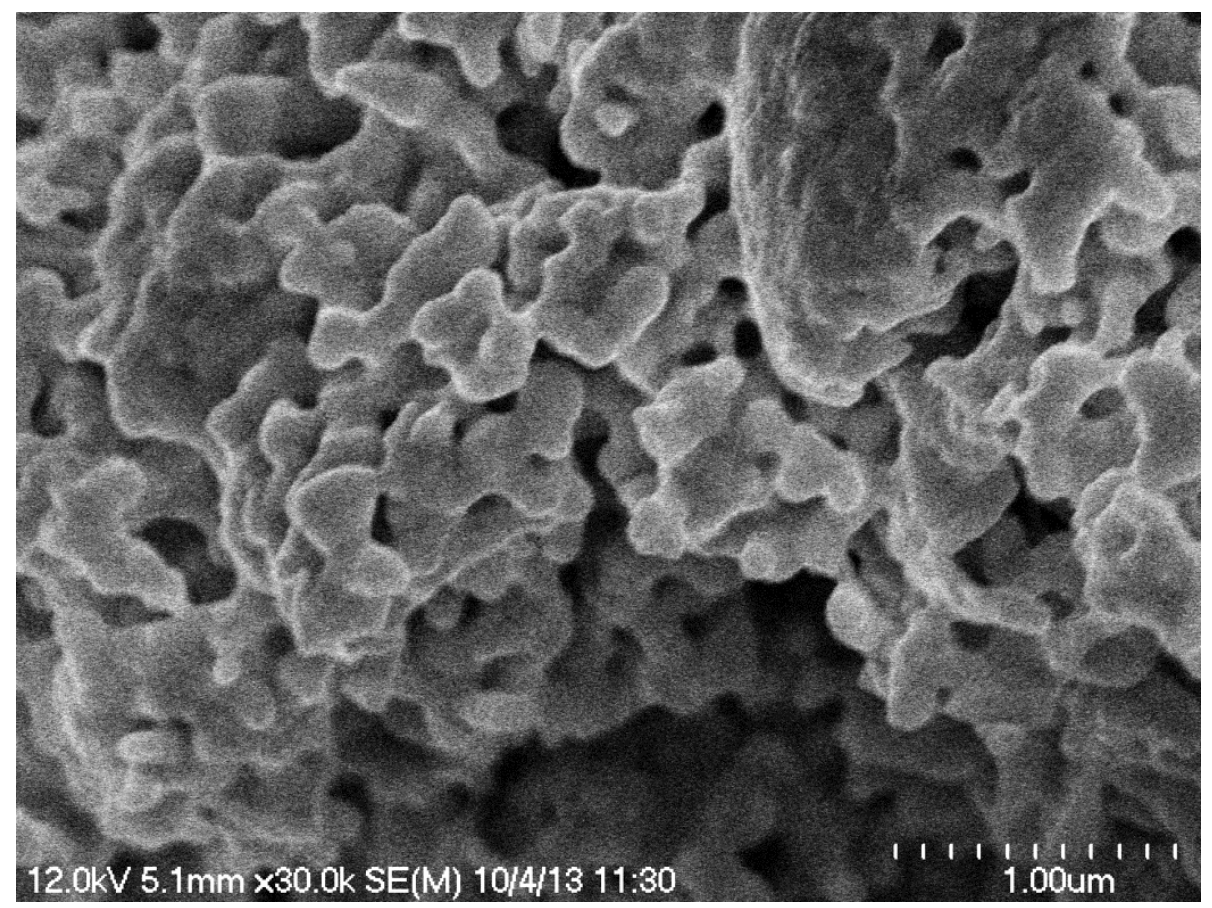

Figure 2-2 30K magnification SEM image of nanoporous gold foams reduced by hydrazine with EG involved 




Figure 2-3 10K magnification SEM image of nanoporous gold foams reduced by hydrazine without EG involved

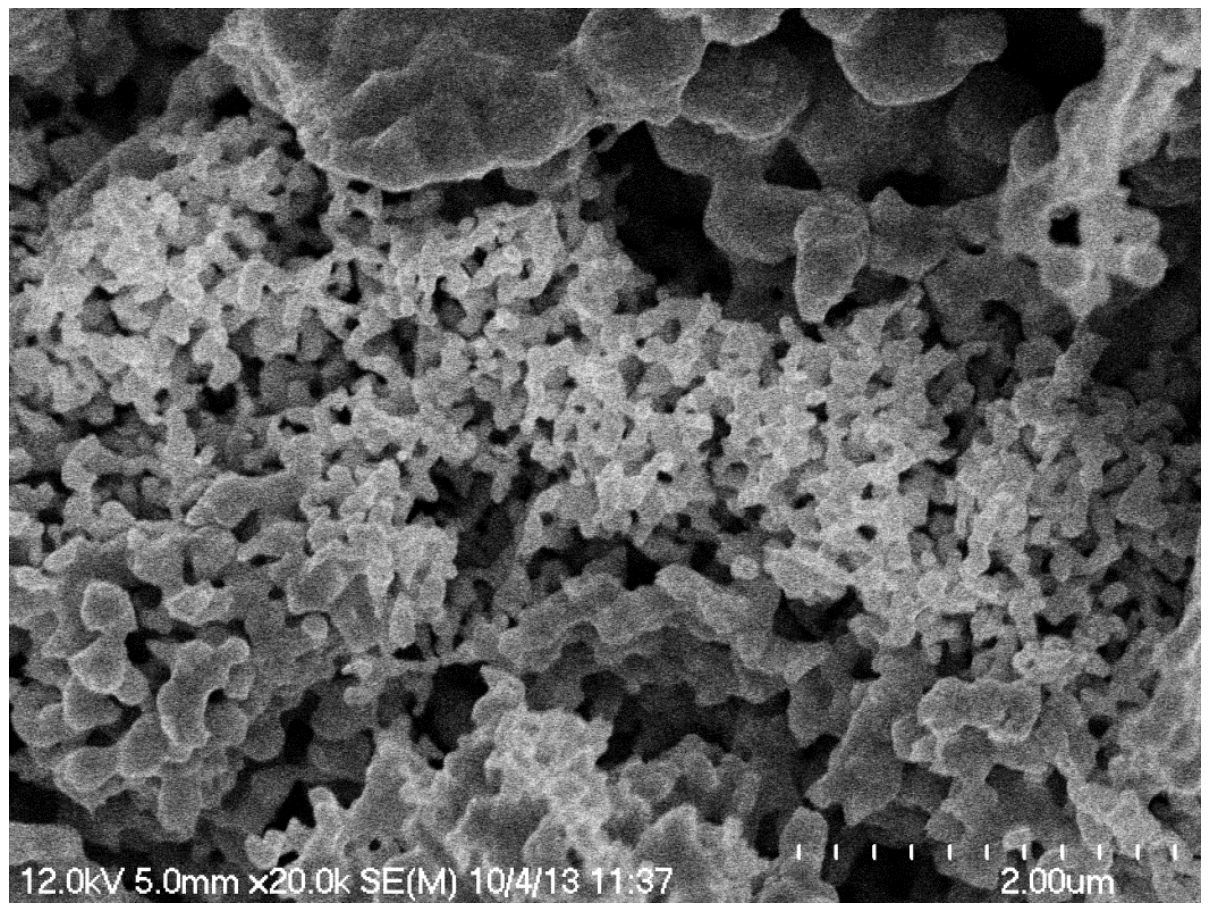

Figure 2-4 20K magnification SEM image of nanoporous gold foams reduced by hydrazine without EG involved 
The size of gold ligament is typically consisted of aggregated particles. The size are various from $1 \mu \mathrm{m}$ to $10 \mu \mathrm{m}$. Without any mechanically gathering, the particles fused together and then fabricated a porous sub-micro structure. The sizes of the constituent fused particles at room temperature are $100 \mathrm{~nm}$ to $500 \mathrm{~nm}$, which is a relatively small size, which also means a relatively higher specific surface area.

Figure 2-1 and 2-3 are images in relatively low magnifications. These SEM images show the mechanical integrity with obvious cracks and the inhomogeneity through the thickness. Figure 2-2 and 2-4 illustrated network structure with a higher magnification, a cross-link porous sub-microstructure can be easily observed. Relatively high porosity is also provided.

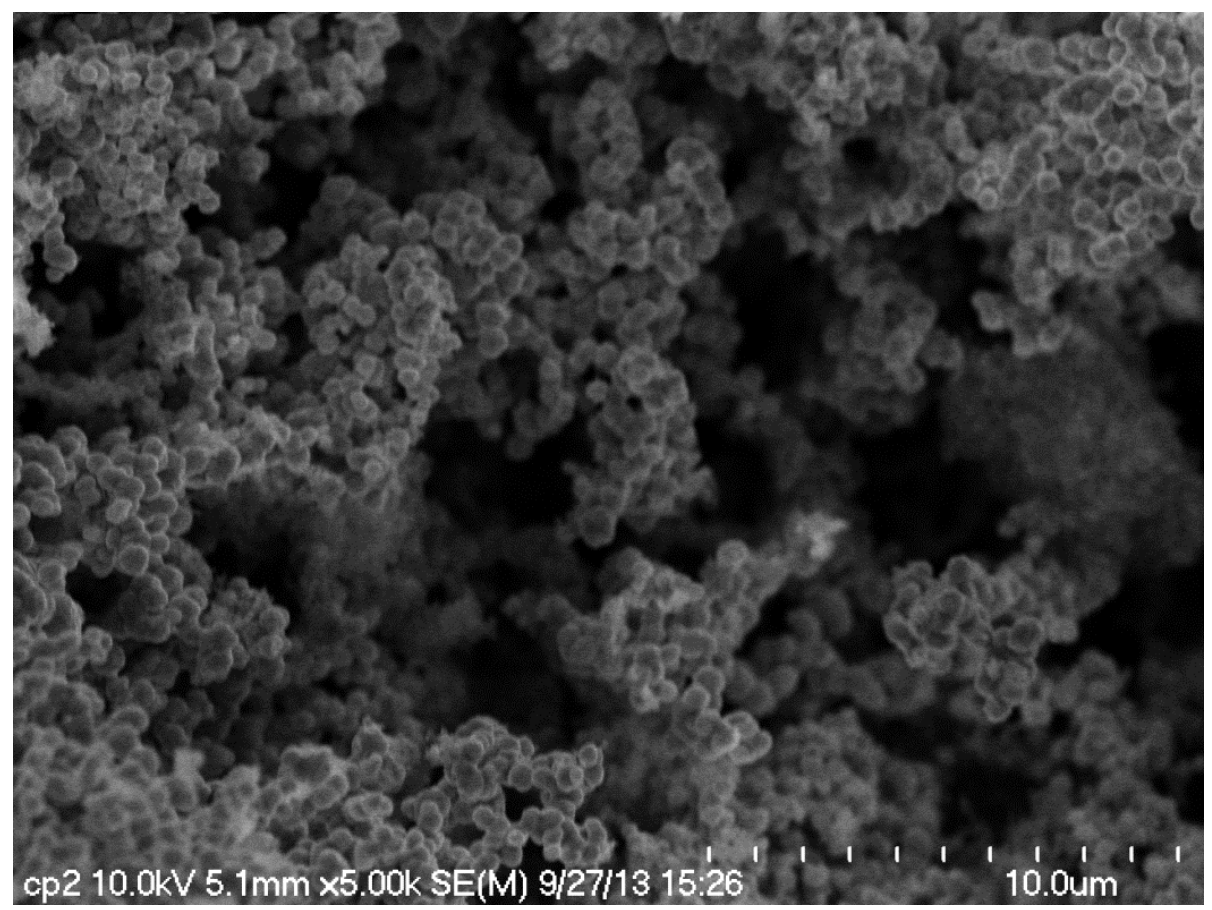

Figure 2-5 5K magnification SEM image of nanoporous nickel foams reduced by hydrazine 


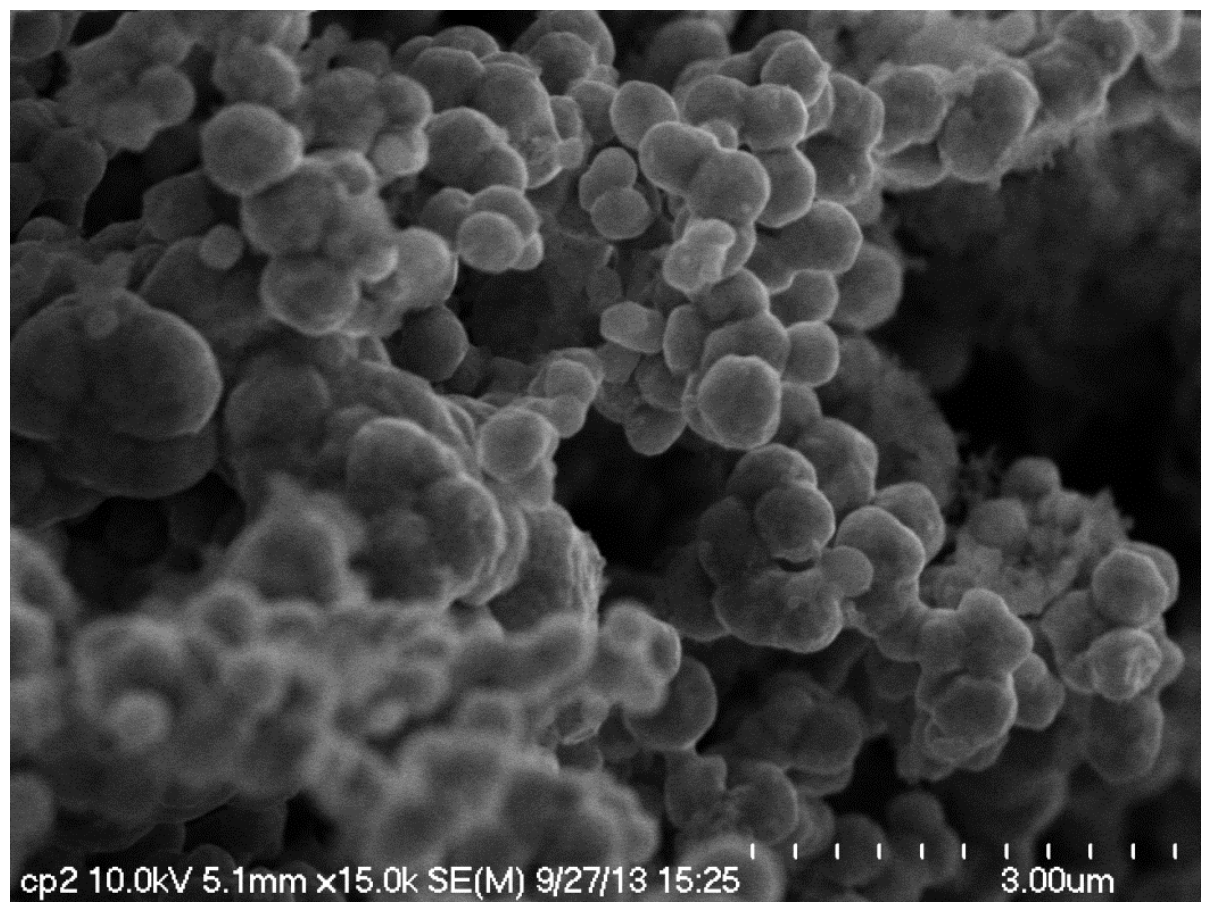

Figure 2-6 15K magnification SEM image of nanoporous nickel foams reduced by hydrazine

Figure 2-5 and 2-6 provide the SEM images of nanoporous nickel foams generated with hydrazine with different magnification. Nickel nano particles are highly dispersed and not homogeneously placed as well. From figure 2-6, the nickel particles are in the size of $3 \mu \mathrm{m}$ with the magnification of $15 \mathrm{~K}$, which is a little bit larger than the particles that construct the nanoporous structure for gold foams.

\subsubsection{Possible Mechanism}

Hydrazine is necessary to not only the reduction process but also the formation of these foam structures in nanoscale in our experiments. Without the hydrazine, metal molecules are not able to be generated from the reduction of metal ions. The $\mathrm{N}_{2}$ released during the reaction is the key factor for the formation of the nano-scale pores in the network structure. According to the previous work, 1D or 2D structures can be 
self-assembled in the present of $\mathrm{N}_{2} \mathrm{H}_{4}$. In this research, 3D structures are constructed with the present of $\mathrm{N}_{2} \mathrm{H}_{4}$.

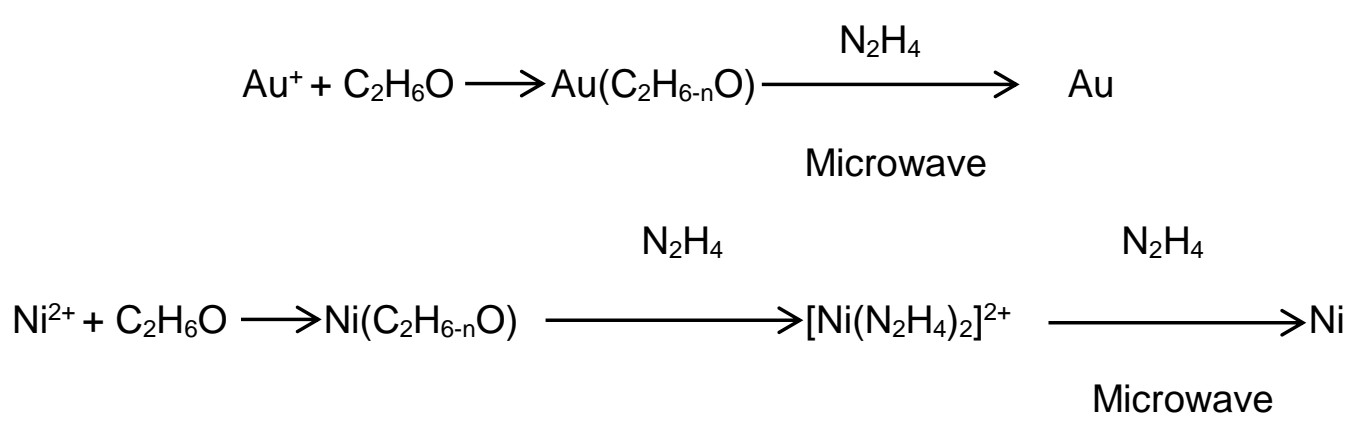

Scheme 1 Proposed chemical reaction mechanisms for hydrazine synthesis

The presence of EG was also important in the experiments to help forming the $3 D$ nanoporous structure. The chelate ligand, $\mathrm{M}\left(\mathrm{C}_{2} \mathrm{H}_{6-n} \mathrm{O}\right)$, was what we desired in the reaction to pretend the metal molecules from gathering too fast. This phenomenon promises the molecules to have adequate time to aggregate into the structure we want. The subsequent addition of hydrazine caused the precipitant of gold metal in the presence of $\mathrm{Au}^{+}$, which can be detected by XRD shortly after the addition. In the case of nickel, after the chelate ligand was formed, hydrazine reacted with the chelate ligand into a complex ion first, which can be indicated by the color of the solution change from yellow to pink. Then with the microwave irradiation, the 3D nanoporous nickel foams would generated with a great amount of gas released at last. All the mechanisms are shown in the Scheme 1. 


\subsection{Borohydride Synthesis}

\subsubsection{Materials}

Gold (I) Chloride (CAS\#10294-29-8, AuCl, 99+\%, MW: 232.42), ethylene glycol (CAS\#107-20-1, C2H6O2, $\geq$ 99\%, MW: 62.07) and ethanol (CAS\#64-17-5, C2H6O, $\geq$ 99.5\%, MW: 46.07) were purchased from ACROS Organics. Nickel (II) Chloride (CAS\#7718-54-9, NiCl2, 98\%, MW: 129.60), was obtained from ALDRICH. Hydrazine monohydrate (CAS\#7803-57-8, H4N2·H2O, $\geq 99 \%$, MW: 50.06) was purchased from Alfa Aesar Chemicals. All materials were used without any further purification.

\subsubsection{Sodium Borohydride Synthesis Process}

Since the generation of the NMFs was due to the redox reaction, Gold and Nickel NMFs were supposed to be produced with the reduction reaction using sodium borohydride as the reductant as well. The same amount of gold chloride as in the hydrazine synthesis was used in this experiment. The gold chloride powders were dissolved in the mixture of $1 \mathrm{ml}$ ethanol and $1 \mathrm{ml} \mathrm{EG}$. Then the mixture was stirred in the microwave vials with a stir bar to obtain a homogenous solution. After a 5 -minute-stirring, $0.015 \mathrm{~g}$ sodium borohydride, the reducing agent, was added into the vial. The solution turned into black as soon as the sodium borohydride was added. At the same time, great amount of bubbles were generated. After stirring for 15 minutes, the solution turned to be clear with small black particles in it. Next step is to heat the solution using microwave oven. The capped vial was sent into the oven and then started heating at $150{ }^{\circ} \mathrm{C}$ for 5 minutes. When the heating process finished, the sample produced needed to be washed by ethanol, centrifuged and dried just the same as the sample generated with 
hydrazine. The final products were preserved in a labeled capped vial. The sample produced with this synthesis method was $0.029 \mathrm{~g}$ from $0.039 \mathrm{~g}$ gold chloride, which means the yield achieve $87.74 \mathrm{wt} \%$.

The borohydride synthesis for nickel NMFs followed almost the same steps as those for gold NMFs except the amount of nickel chloride used was determine to be $0.026 \mathrm{~g}$, in order to guarantee the same ion concentration. Since the reaction was much more vigorous, a huge amount of gas was already released during the stirring process, which helped to avoid the pressure problem occurring in the hydrazine synthesis process of $\mathrm{Ni}$ NMFs. However, there was another problem with this synthesis. The yield of the synthesis was much lower than any other synthesis we reported above. We obtained $0.011 \mathrm{~g}$ sample from $0.09 \mathrm{~g}$ nickel chloride. The yield corresponding to it was 27.00 $\mathrm{wt} \%$.

\subsubsection{Results and Discussions}

Figure 2-7 -2-10 show the porous structure of the NMFs produced with the borohydride synthesis. The 3D porous networks are still clear with the SEM images for nanoporous gold foams, which means the borohydride synthesis works well with the microwave irradiation. Although the ligaments were not uniformly sized, this also implied that the formation of the network structure was due to the aggregation of nanoparticles. The particles that aggregated to form the network had the size of $300 \mathrm{~nm}$ to $1000 \mathrm{~nm}$. And the pores are in the scale of $500 \mathrm{~nm}$ to $2 \mu \mathrm{m}$. Compared with the samples produced with hydrazine, the sizes of the ligament as well as the sizes of the pores were even smaller. 


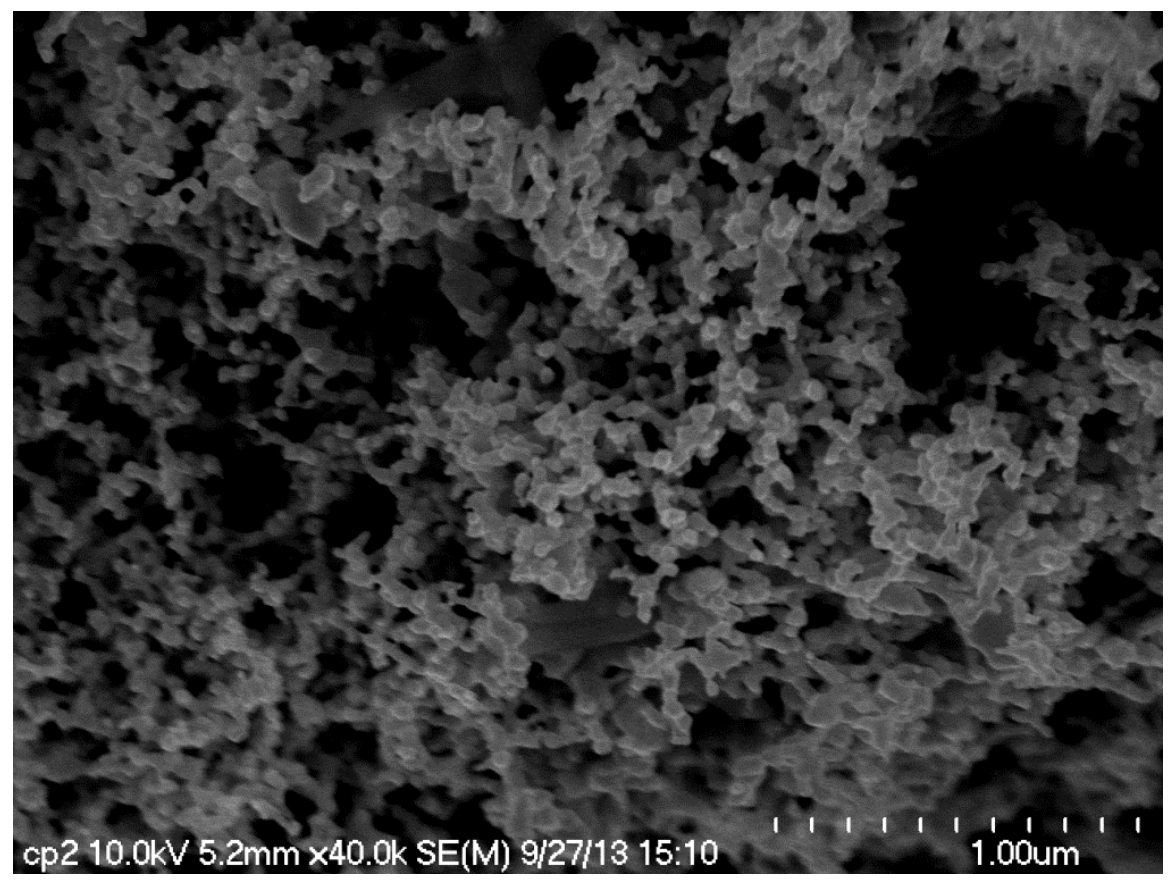

Figure 2-7 40K magnification SEM image of nanoporous gold foams reduced by sodium borohydride with EG involved

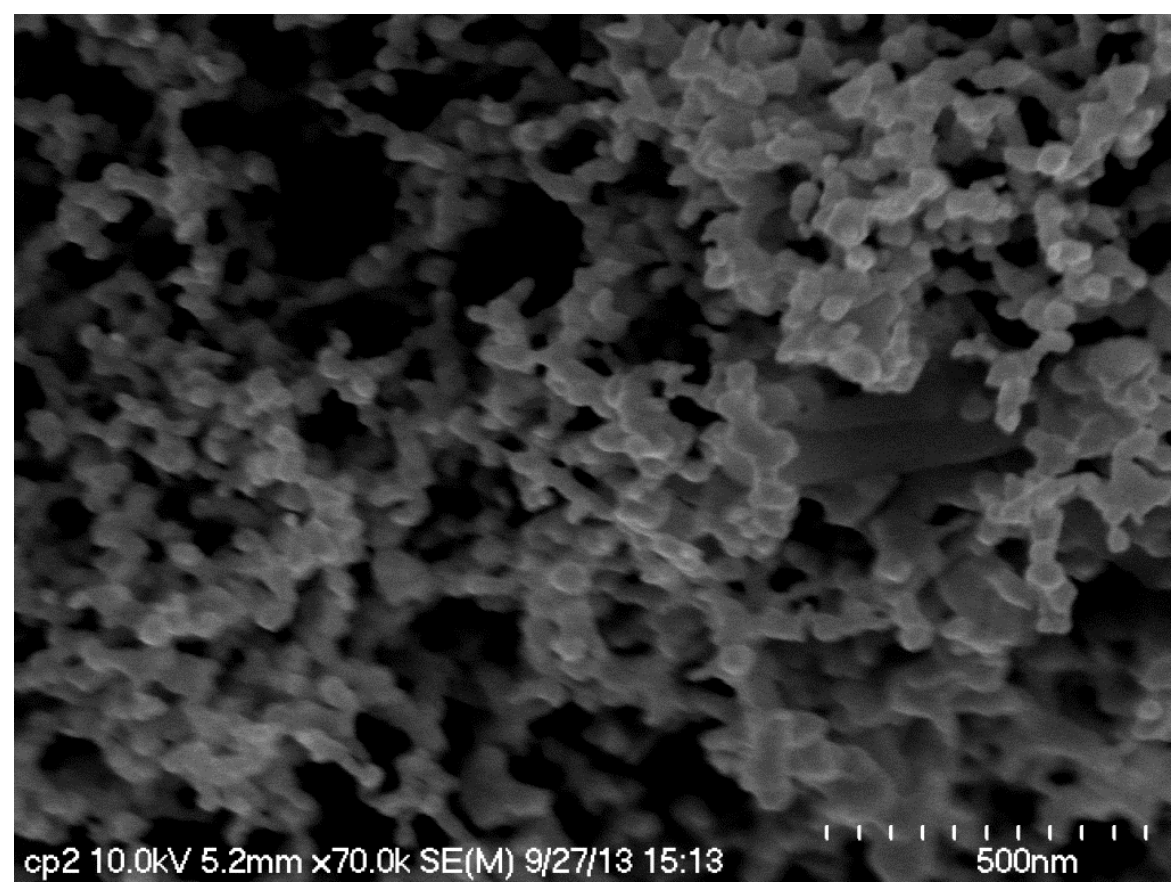

Figure 2-8 70K magnification SEM image of nanoporous gold foams reduced by sodium borohydride with EG involved 


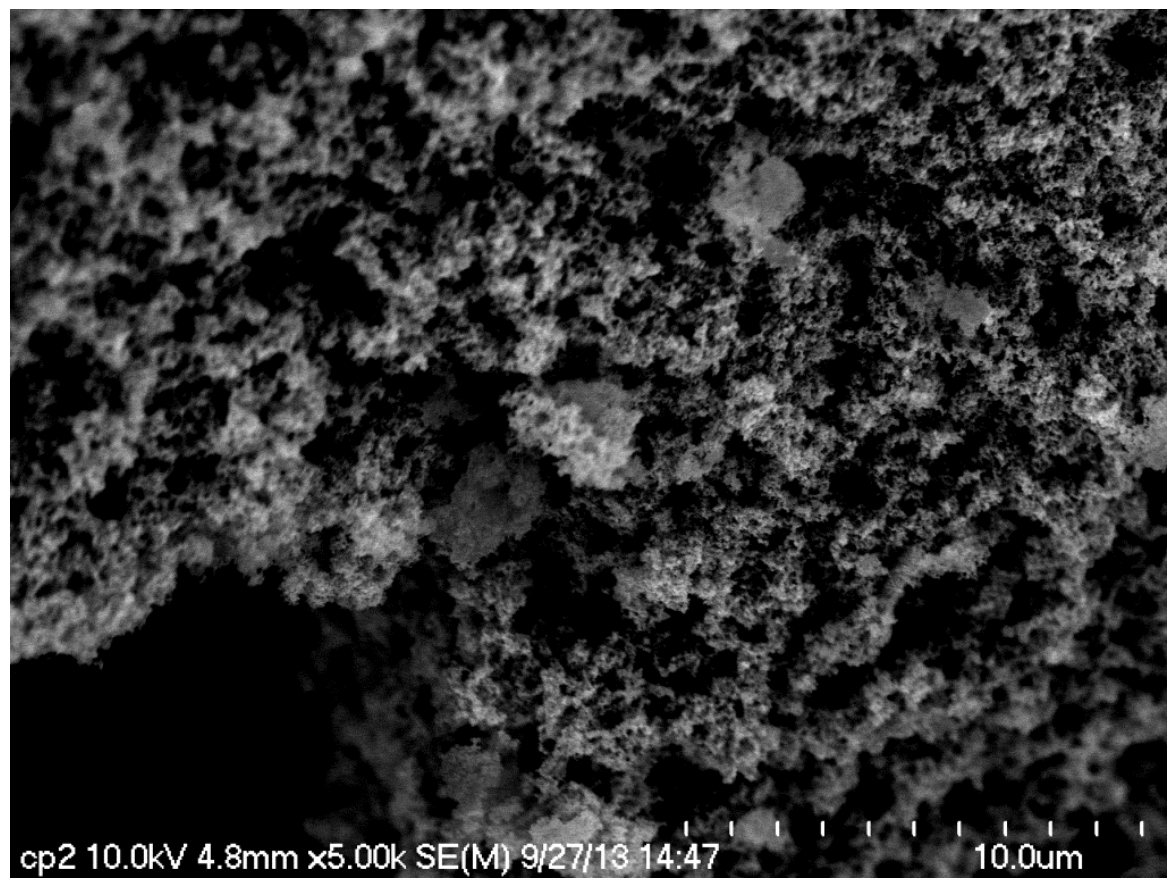

Figure 2-9 5K magnification SEM image of nanoporous nickel foams reduced by sodium borohydride

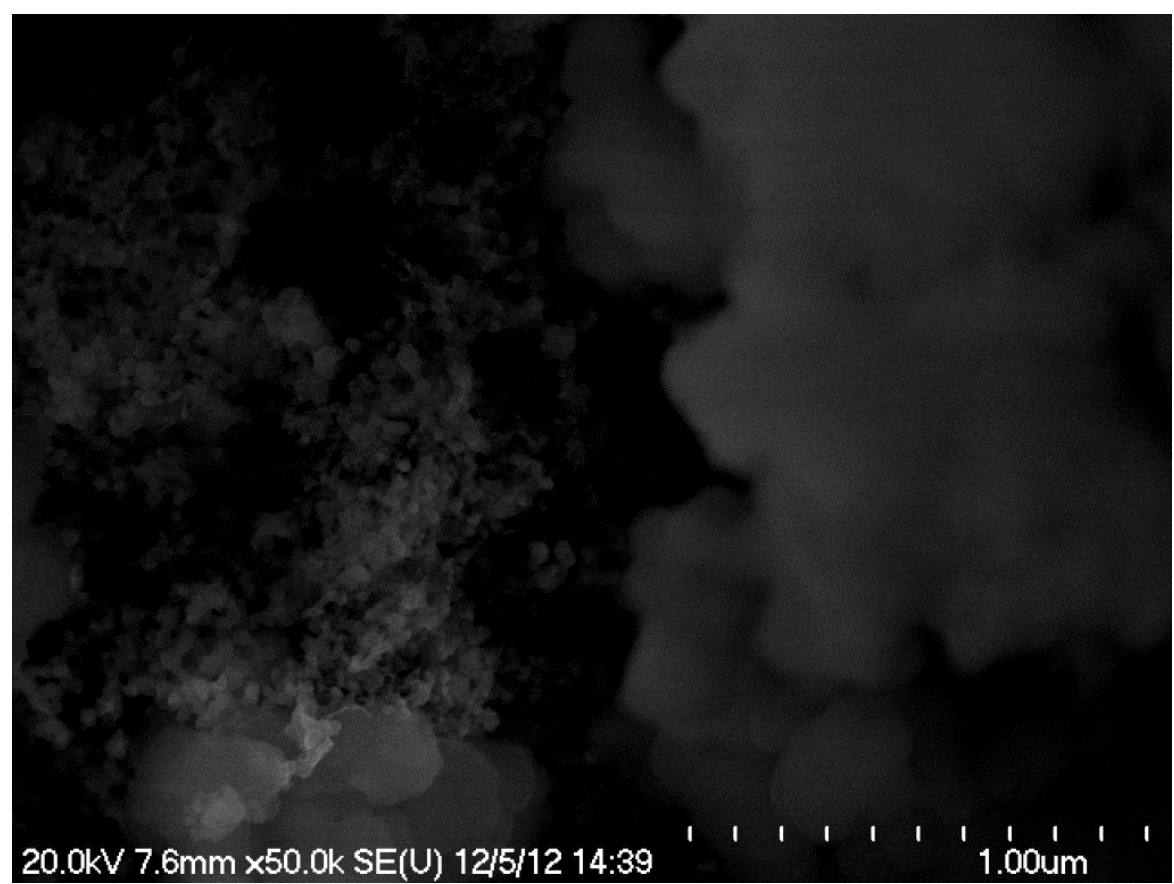

Figure 2-10 50K magnification SEM image of nanoporous nickel foams reduced by sodium borohydride

Even though the SEM images were at a relatively lower magnification, the 3D foamstructure was able to be observed. And obviously the size of the pores and the 
ligaments were at the scale of nanometer. As a result, the nanoporous nickel foams were able to be generated with the borohydride synthesis. But the products did not have the uniform thickness. With a higher magnification, the particles that constructed the ligaments and the pores were of the size ranging from $500 \mathrm{~nm}$ to $3 \mu \mathrm{m}$.

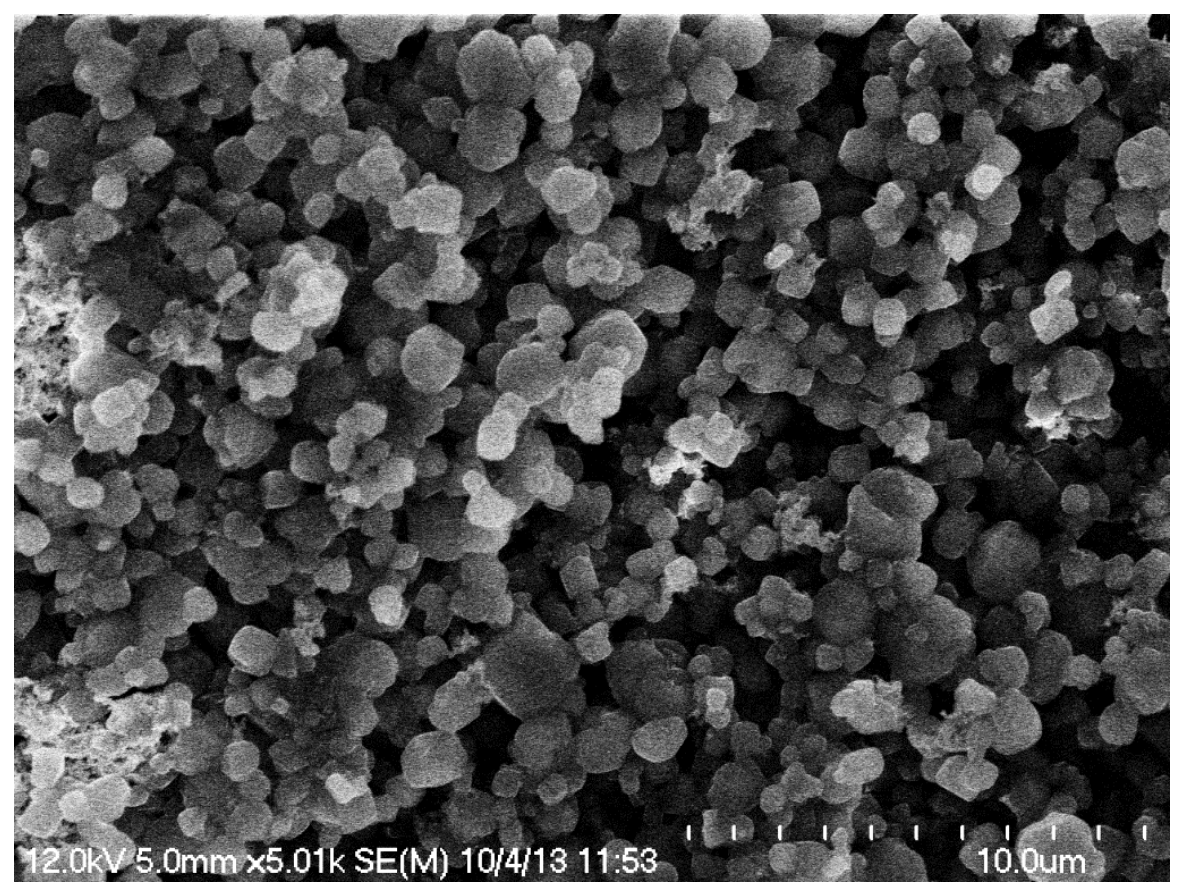

Fig 2-11 5K magnification SEM image of nanoporous gold foams reduced by sodium borohydride without EG

Figure 2-11 and 2-12 present the structure details of the samples produced with sodium borohydride as reducing agent and no ethylene glycol involved. According to the SEM image with magnification of $5 \mathrm{~K}$, the structure of the products were more likely a particlestructured rather than foam-structured. The particles grew up to 10 to $30 \mu \mathrm{m}$ in diameter. However, some 3D nanoporous structures were also captured by SEM. The pores and the particles of ligament were also in the scale of nanometers. But such structures only appeared in the interspace of the particles and occupied a low volume fraction of the samples. The reason for this phenomenon might be the absence of EG. EG was used to form the chelate ligand in order to prevent the metal molecules 
aggregating too fast, which results in the nanoparticles instead of the nanoporous foams.

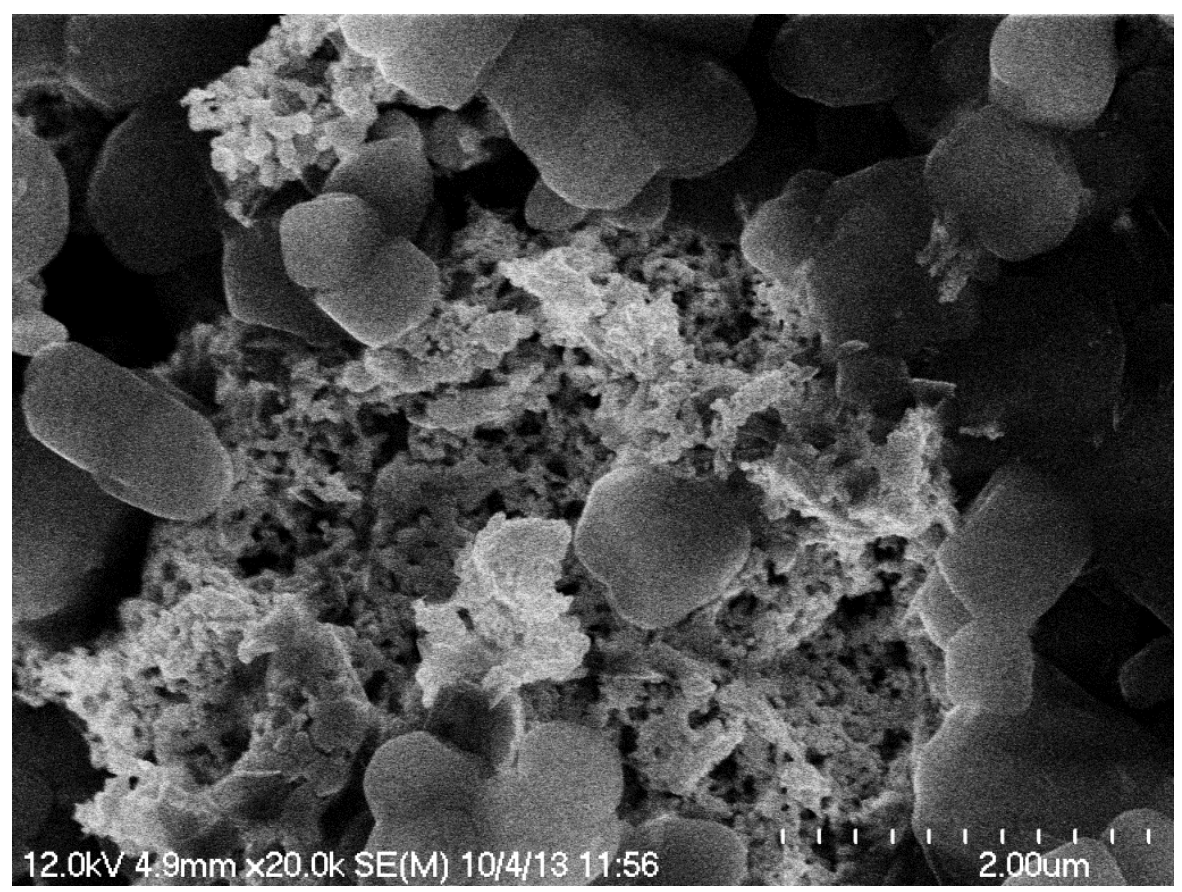

Figure 2-12 20K magnification SEM image of nanoporous gold foams reduced by sodium borohydride without EG

\subsubsection{Possible Mechanism}

The redox reaction shown in the scheme 2 illustrates the possible mechanism of the sodium borohydride synthesis process.

$$
\mathrm{M}^{+}+\mathrm{NaBH} 4 \rightarrow \mathrm{M}+\frac{1}{2} \mathrm{H} 2+\frac{1}{2} \mathrm{~B} 2 \mathrm{H} 6+\mathrm{Na}^{+}
$$

$M$ stand for the metal used in the process

Scheme 2 Proposed redox reaction mechanisms for borohydride synthesis

Metal molecules will be reduced from the metal ions by the presence of the sodium borohydride. Sodium borohydride is not only the reducing agent, the hydrogen released during the redox reaction is also the key factor to form the nanoporous structure we proposed to obtain. The borohydride anions were adsorbed onto the nanoparticles and 
the presence of EG prevented the aggregation of particles. The desired nanoporous foams can be produced through the microwave irradiation heating. 


\section{CHAPTER 3}

\section{APPLICATIONS OF NANOPOROUS METAL FOAMS}

\subsection{Gold NMFs application for SERS}

\subsubsection{Background}

Raman scattering was firstly reported by Fleischmann, Hendra and McQuillan in 1973. (Fleischmann, Hendra et al. 1974) The original idea was to generate a high surface area on the roughened metal. In 1977 two groups independently noted that the concentration of scattering species could not account for the enhanced signal and each proposed a mechanism for the observed enhancement. One is the electromagnetic effect proposed by Jeanmaire and Van Duyne. The other is the charge transfer effect proposed by Albrecht and Creighton. (Albrecht and Creighton 1977; Jeanmaire and Van Duyne 1977) Both of the theories are accepted as explaining the SERS effect.

SERS was observed primarily for analytes adsorbed on to $\mathrm{Au}, \mathrm{Ag}$ and $\mathrm{Cu}$, and $\mathrm{Li}, \mathrm{Na}$ and K. Any metal would be able to exhibit surface enhancement theoretically, but the metals above satisfy calculable requirements and provide strongest enhancement. Attributed to the stability, $\mathrm{Au}$ and $\mathrm{Ag}$ are generally used as the substrates for SERS. $10^{2}-10^{3}$ of enhancements are also available with $\mathrm{Pd}$ and $\mathrm{Pt}$ as the substrates.

With the development of nanotechnology, various nanostructures improved the ability of surface enhancement, such as 2D Au nanomushroom arrays (Naya, Tani et al. 2008) and shell-isolated nanoparticles(Li, Huang et al. 2010) Other materials are also capable 
of being the substrates for SERS in recent years with or without the help with gold or silver, including carbon nanotubes coated by Au nano particles film(Zhang, Chen et al. 2012) and gold/silver coated nanoporous ceramic membranes. (Kassu, Robinson et al. 2010)

As mentioned before, gold nanostructures performed excellent as a substrate. Many different analytes were mentioned such as rhodamine $6 \mathrm{G}$ and 1,2-benzenedithiol. In this work, R6G was used as an analyte. Different limit of detections were reported from many previous work, for instance, Andrzej Kudelski showed his work to reduce the concentration of $\mathrm{R} 6 \mathrm{G}$ to $10^{-8} \mathrm{M}$ by an electrochemically roughened silver substrates.(Kudelski 2005) Yu Chuan Liu and coworkers reported that the detection limit was successfully reduced by six orders of magnitude from $2 \times 10^{-9} \mathrm{M}$ to $2 \times 10^{-15} \mathrm{M}$. (Liu, Yu et al. 2006) Dar Nitzan et al. also reported that the detection limit of R6G can be achieved to $10^{-12} \mathrm{M}$ by the composite structure made of $\mathrm{Ag}$ nanoparticles adsorbed on GaN nanowires.(Dar, Wen-Jing et al. 2011) Moreover, Selena Chan et al. reported a detection limit of $10^{-4} \mathrm{M}$ on small molecules from silver-coated silicon nanopores substrate using $785 \mathrm{~nm}$ excitation. (Chan, Kwon et al. 2003) Ravula Thirupathi and coworkers reported a detection limit of $1 \mathrm{mM}$ on a gold microrods. (Thirupathi and Prabhakaran 2011)

\subsubsection{Experimental Section}

I-Raman microscope and spectrometer from B\&W Tek Inc. was the instrument used through this process. A key was turned clockwise 90 degrees to start the system at first. A probe was opened by slide the switch and then located close to the sample. Computer software programmed for the observation was initialed to find the Raman 
signal. Next step was to relocate the probe to maximize the focal point of the laser beam on the sample by observing the Raman signal on the computer screen. The regulator on the screen was treated as the indicator of the ideal focal point. When the regulator is placed against the sample accurately, the ideal focal point is achieved. A dark scan should be taken as the background, in order to eliminate the influence of other light sources in the environment and obtain a lower noise of the instrument. After setting up the exposure time and the laser power, the sample could be scanned by the instrument and a Raman spectrum would be reported by the software.

In this research, prepared gold NMFs were tested for SERS activity. R6G was used as the analyte to figure out the lowest limit of detection with the presence of silver NMFs. R6G (CAS\#989-38-8, C28H31N2O3Cl, 99\%, MW: 479.01), was obtained from SigmaAldrich. $5 \mathrm{mg}$ gold NMFs were immersed in $0.2 \mathrm{ml} \mathrm{R6G}$ solution for 36 hours for a better detection. Then the gold NMFs were taken out of the solution and put on a glass slide for observation. Raman spectra were recorded at room temperature with a $785 \mathrm{~nm}$ laser as light source. The characteristic signals for R6G were enhanced multifold when observed over the gold NMFs substrate.

\subsubsection{Result and Discussion}

Figure 3-1 shows the Raman Spectra of R6G molecules on the surface of gold NMFs. The exciting radiation was a laser with wavelength of $785 \mathrm{~nm}$. The gold NMFs were firstly taken out with a spatula and settled on the surface of a glass slide for the observation. In order to prevent the noise of water, we had to wait for 5 minutes for the 
water to evaporate. Of course, not all the water was evaporated at the same time. We could observe from the screen that the boundary between wet and dry regions eliminated rapidly towards the metal foams. Since the size of R6G molecule is around $1.4 \mathrm{~nm}$, we assumed that in the densely packed R6G monolayer a single molecule would not take more than $4 \mathrm{~nm}^{2}$ in area. The detection limit of $5.2 \times 10^{-7} \mathrm{M}$ of $\mathrm{R} 6 \mathrm{G}$ is lower comparing to previous works, such as $1 \mathrm{mM}$ reported by Ravula Thirupathi and coworkers. (Thirupathi and Prabhakaran 2011) Compared with the previous work in our lab, the silver NMFs could be the substrate as well in order to achieve the detection limit of 2x10-6 M, which was a bit higher than use the gold NMFs generated in this research. But there are lower detection limit reported as well with other gold substrates or different treatment of surface.

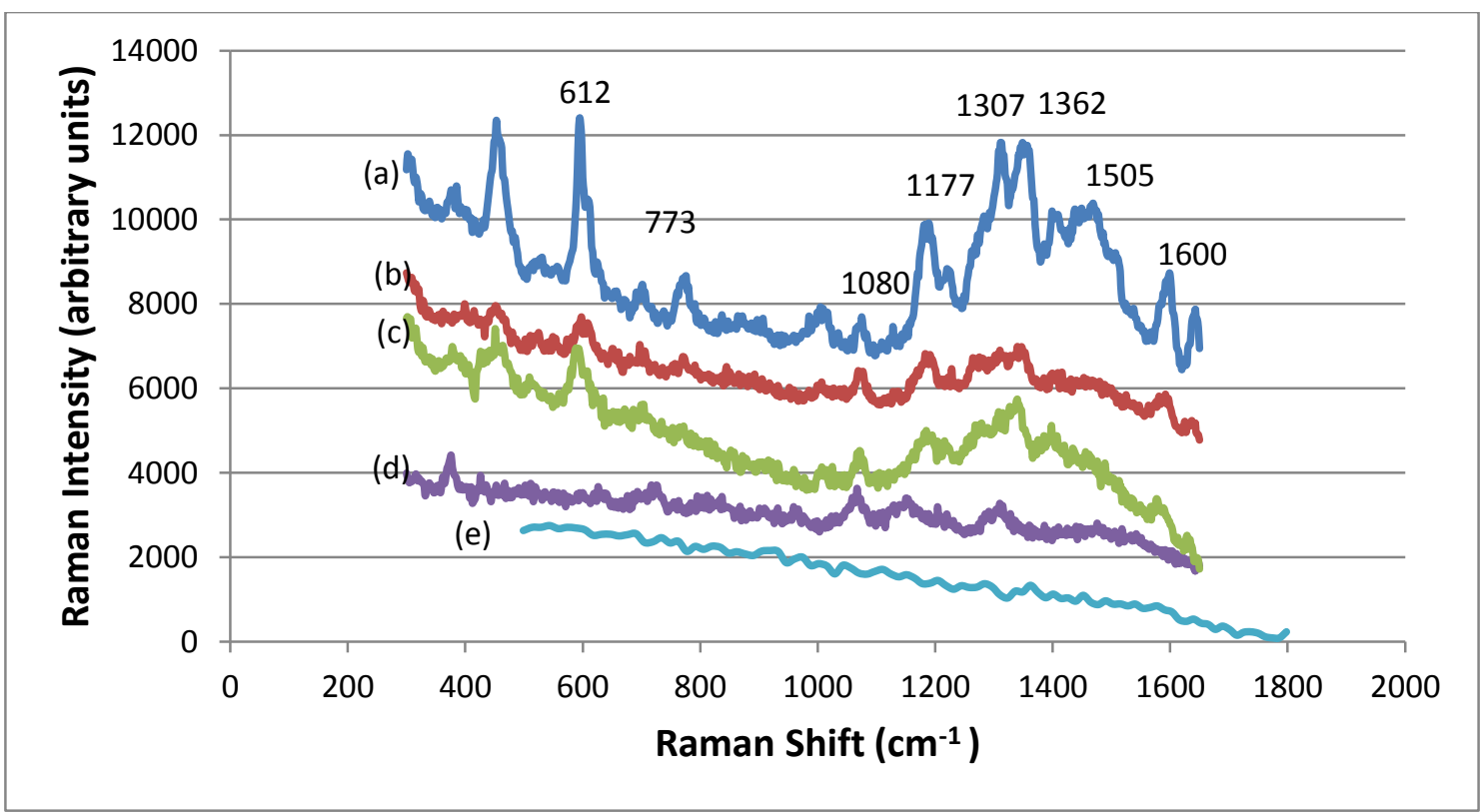

Figure 3-1 Raman spectra of R6G molecules deposit on surface of gold NMFs: (a) $1.0 \times 10^{-5} \mathrm{M}$; (b) $2.1 \times 10^{-6} \mathrm{M}$; (c) $1.0 \times 10^{-6}$ $\mathrm{M}$; (d) $5.2 \times 10^{-7} \mathrm{M}(\mathrm{e}) 1.0 \times 10^{-8} \mathrm{M}$. The spectra have been scaled and vertically shifted to enhance the clarity of the presentation. 
The prominent features at $612,773,1177,1307,1362$ and $1507 \mathrm{~cm}^{-1}$ were observed, in agreement with previous experimental and theoretical investigations.(He, Gao et al. 2012) The peak at 612 is due to the $C-C-C$ ring in-plane bending remains unaffected from the dye-host interactions. A peak at $773 \mathrm{~cm}^{-1}$ was observed for the $\mathrm{C}-\mathrm{H}$ out-of plane bending of both condensed rings are degenerated in the solution. And other bands were observed in the higher wavelength region. The peaks at 1307, 1362 and $1505 \mathrm{~cm}^{-1}$ were obtained due to the aromatic C-C stretching modes. These modes gain intensity due to Franck-Condon overlap integrals, which depends on the extent of displacement of the excited state potential well along the normal coordinate (Saini, Kaur et al. 2005; He, Gao et al. 2012)

\subsection{Nickel NMFs application for Degradation of MO}

\subsubsection{Background}

As mentioned in Chapter 1, azo dyes have been regarded as environmental pollution necessary to be dealt with in the recent years. Various catalysts, especially the photocatalysts, are developed and engineered to degrade the azo dyes. With the properties such as stable and non-toxic in air and water, nickel NMFs are selected as an ideal catalyst for MO degradation at room temperature.

Different form the bimetallic nano-products that already used in the procedure of degradation of $\mathrm{MO}$, nanoporous metal foams have the unique characteristics like defective and strained surface. According to the research of Masataka Hakamada et al, the defective and strained surface as well as the large surface area of the NMFs 
significantly enhanced the rate of the degradation reaction. (Hakamada, Hirashima et al. 2012) Otherwise, some other groups also reported that bimetallic nano-materials improve the rate of degradation to $10 \%-15 \%$ and reduce the concentration to a lower level that single metal due to the enhanced intermolecular effect.

\subsubsection{Experimental Section}

Solid methyl orange (CAS\#547-58-0, (CH3)2NC6H4:NC6H4SO3N2, 90\%, MW: 327.33) from Fisher Scientific Company was used in our research. $0.328 \mathrm{~g}$ solid MO was dissolved in $500 \mathrm{ml}$ water in order to obtain the $\mathrm{MO}$ solution with concentration of $2 \times 10^{-3}$ $\mathrm{mol} / \mathrm{L}$. Solution with lower concentration, including $5 \times 10^{-4}$ and $2 \times 10^{-5} \mathrm{~mol} / \mathrm{L}$, was obtained by diluting the initial solution. In the experiments, $0.025 \mathrm{~g}$ nickel NMFs produced with the hydrazine synthesis was added into $5 \mathrm{ml} \mathrm{MO}$ solution and the degradation reaction then started spontaneously and immediately at room temperature. All the MO solutions were kept in capped vials in a dark environment to ensure the degradation was due to the catalytic behavior of nickel NMFs rather than the photocatalytic behavior observed with $\mathrm{TiO}_{2}$ and $\mathrm{ZnO} .0 .03 \mathrm{ml}$ of nickel immersed $\mathrm{MO}$ solution was extracted for absorbance analysis at different submersion time. The decrease in concentration and limitation of nickel catalyst could be determined referring to the result of absorbance analysis. A 48-well UV-Vis plate reader was applied for the sample detection. The decay of all the absorbance was observed at the wavelength of $465 \mathrm{~nm}$.

\subsubsection{Results and Discussion}

Obvious decrease of absorbance peak shows the nickel NMFs can serve as an effective catalyst for MO degradation. In general, 15 hours is necessary for the 
absorbance to reduce to $17 \%$ as the initial concentration. Figure $3-2,3-3$ and $3-4$ present the UV-Vis spectra for different MO solutions with different initial concentration. Every curve was obtained with the data for every specific time. For Figure 3-2, the curve having the highest peak stands for the absorbance curve against the wavelength of the initial solution. And the following curves are those collected at 0.5 hour, 0.75 hour towards 26 hours. The peaks are the maximum absorbance of the solution at the specific time point. So the spectra show that the absorbance of MO solution, whose initial concentration was $2 \times 10^{-3} \mathrm{~mol} / \mathrm{L}$, decreased from 3 arb. unit to 0.25 arb. unit after 26-hour-immersion of nickel NMFs.

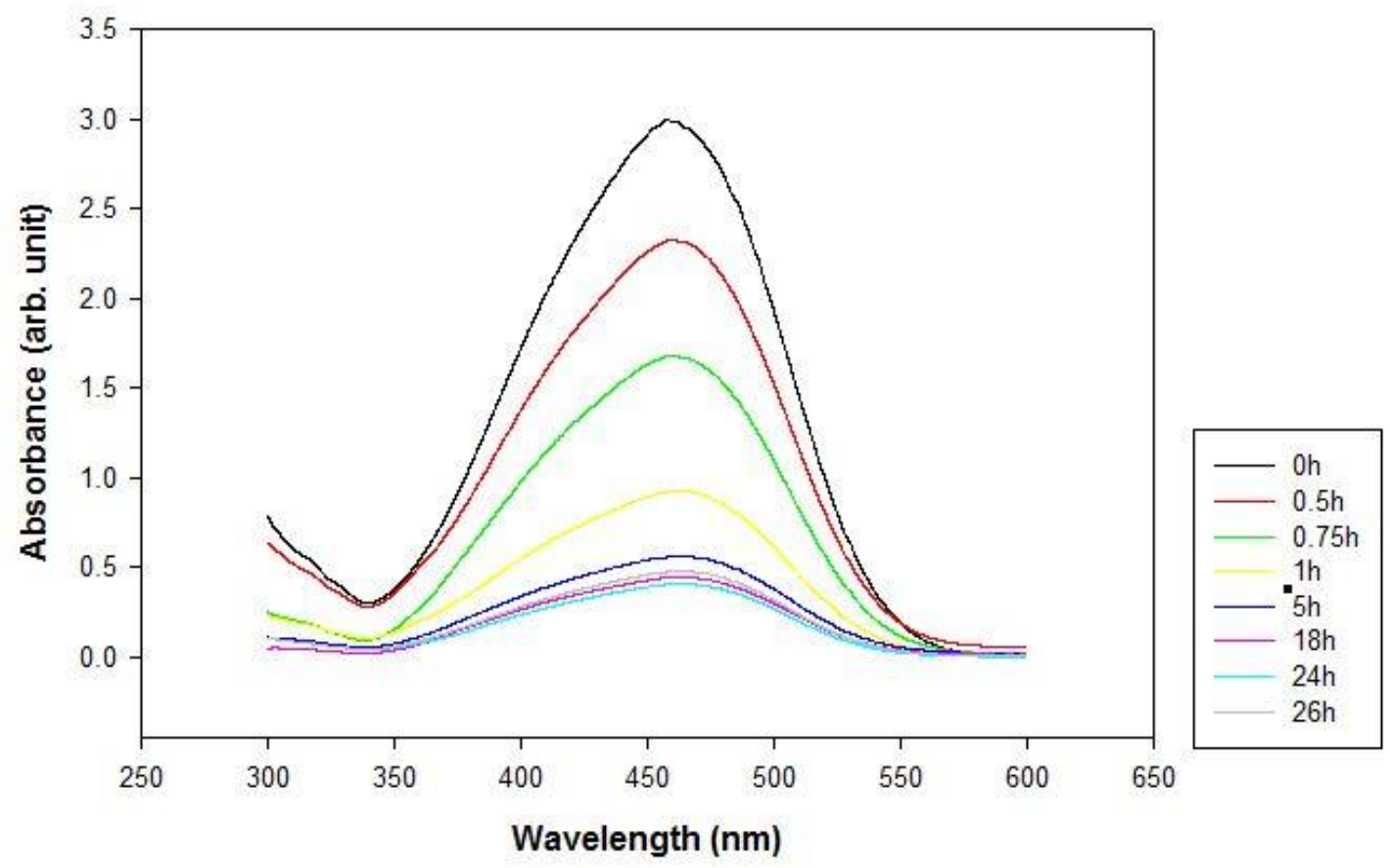

Figure 3-2 UV-Vis spectra of the MO solution $\left(2 \times 10^{-3} \mathrm{~mol} / \mathrm{L}\right)$ after different submersion time at room temperature. 


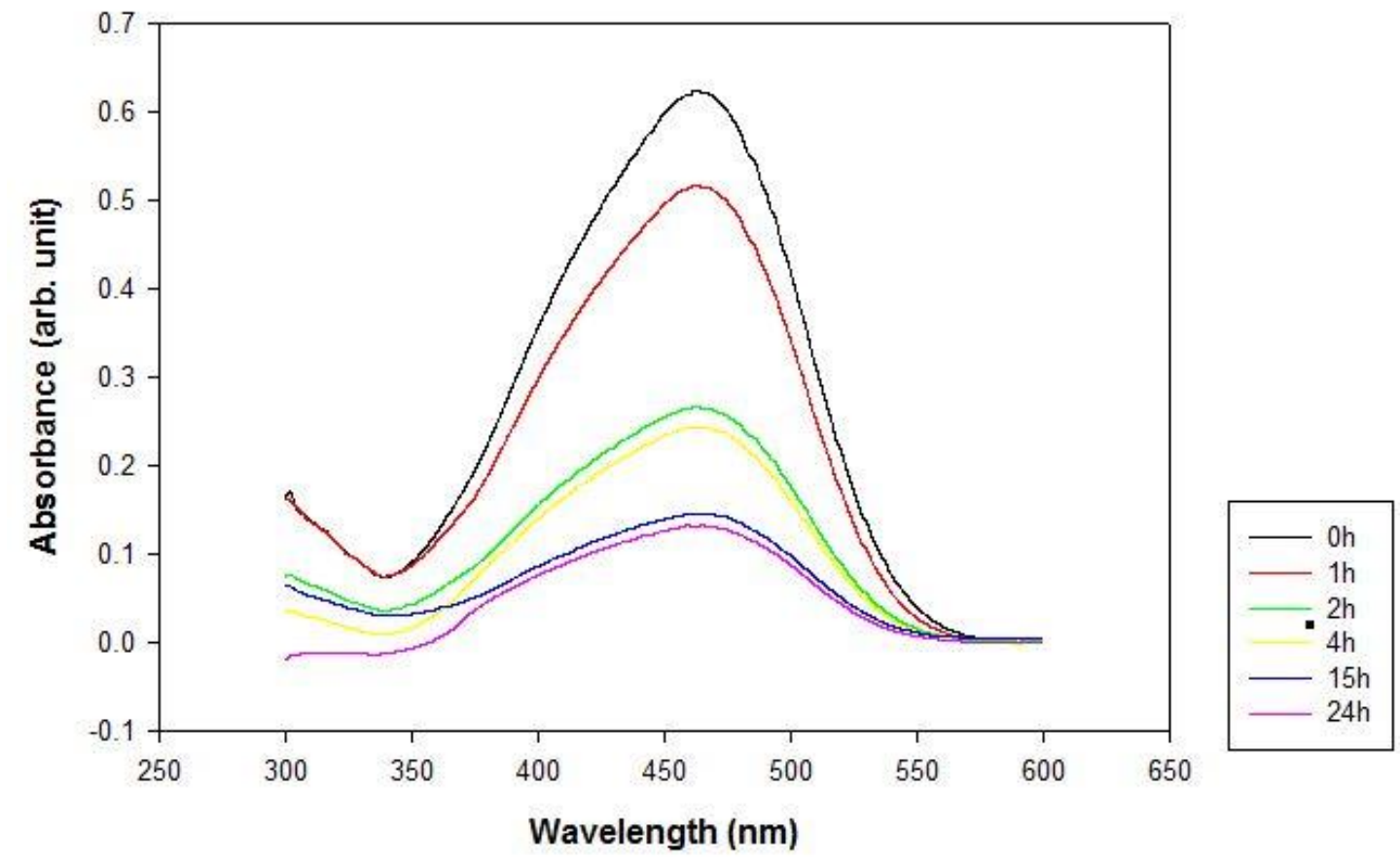

Figure 3-3 UV-Vis spectra of the MO solution $\left(5 \times 10^{-4} \mathrm{~mol} / \mathrm{L}\right)$ after different submersion time at room temperature.



Figure 3-4 UV-Vis spectra of the MO solution $\left(2 \times 10^{-5} \mathrm{~mol} / \mathrm{L}\right)$ after different submersion time at room temperature. 
The same analysis procedure was applied to the spectra presented in the Figure 3-3 and Figure 3-4 as well. In the case of the MO solution with initial concentration of $5 \times 10^{-4}$ $\mathrm{mol} / \mathrm{L}$, the peaks decayed from 0.9 to 0.7 in 1 hour and then continued to 0.25 in 2 hours. After 24 hours, the peak decreased to around 0.1. In the case of the MO solution with initial concentration of $2 \times 10^{-5} \mathrm{~mol} / \mathrm{L}$ the peaks decayed even more rapidly. The absorbance decreased from 0.9 to 0.55 in only 20 minutes and achieved at about 0.15 in 2 hours.

All the data for the spectra were collected by the UV-Vis Microplate Spectrophotometer. Every single line corresponds to a set of data collected for a specific immersion time period. It can be easily figured out that the intensity peaks appeared at the wavelength of $460 \mathrm{~nm}$ to $470 \mathrm{~nm}$. The intensity of the azo dye solution in the visible light region is caused by an azo group ( $(\mathrm{N}=\mathrm{N}-)$. MO contains two phenyl rings bridged by an azo group in its chemical structure, therefore it is surmised that $\mathrm{MO}$ decomposed to single phenyl rig compounds in the presence of nickel NMFs, which reflected as the decoloration of the solution.

In order to discover the time variation of the MO concentration after immersion of nickel NMFs, the ratio of the concentration at specific time and the initial concentration was calculated. Referring to Beer-Lambert Law, the concentration of the solution at specific time can be obtained with absorbance, pathway length and absorptivity, as shown with Equation 1.

$$
C=A /(b \varepsilon)
$$


In the equation, $C$ stands for the concentration in $\mathrm{mol} / \mathrm{L}, \mathrm{A}$ is the absorbance, $\varepsilon$ is the absorptivity with unit of $\mathrm{L} \mathrm{mol}^{-1} \mathrm{~cm}^{-1}$, depending on the compound, and $\mathrm{b}$ stands for the pathway length of the liquid in the cuvette. In the experiment, we took same volume of solution into the cuvettes with same diameter, which promised the pathway length to be the same for all the tests. Since the compounds of the solution were the same, the $\varepsilon$ was a constant. We can conclude that the ratio of the concentration is proportional to the ratio of the absorbance, which shown in the Equation 2.

$$
\frac{C}{C_{0}}=\frac{A}{A_{0}}
$$

Figure 3-5 shows the time variation of $\mathrm{MO}$ concentration after the immersion of the nickel NMFs. Significant decrease in concentration is achieved after the 500-minuteimmersion. $80 \%$ of the $\mathrm{MO}$ was reduced after 500 minutes for the concentration of $2 \times 10^{-}$ ${ }^{5} \mathrm{M}$ and $2 \times 10^{-3} \mathrm{M}$. For the $5 \times 10^{-4} \mathrm{M}$ solution, $70 \%$ decrease can be achieved after the same time period. However, combined with Figure $3-4$, the spectra for the $2 \times 10^{-5} \mathrm{M}$ sample, 1 hour is adequate for the MO concentration reduced to about $20 \%$, which is a rapid process comparing with our other solutions. 


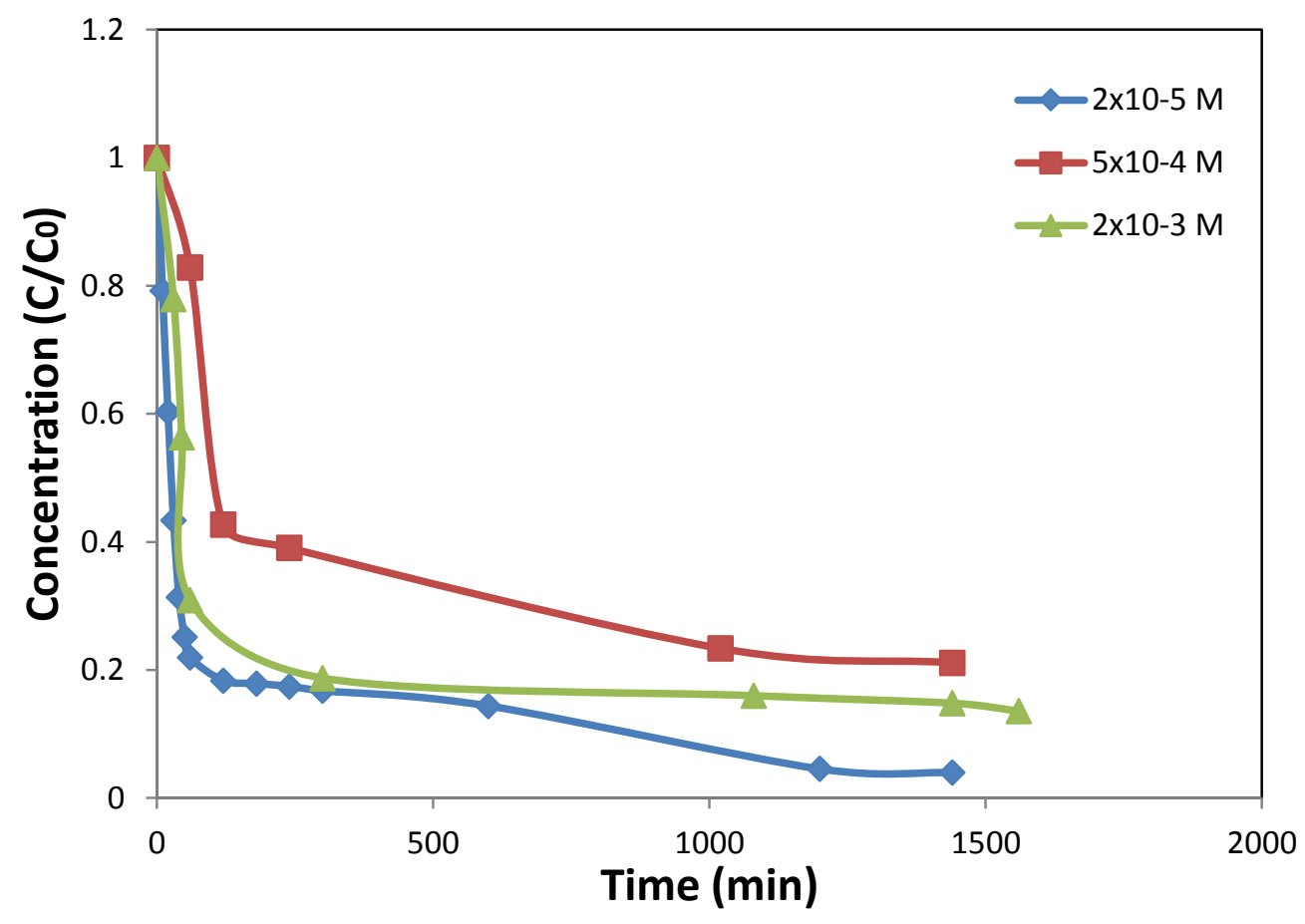

Figure 3-5 Time variation of MO concentration after immersion of nickel NMFs, different concentration of MO solution were also provided.

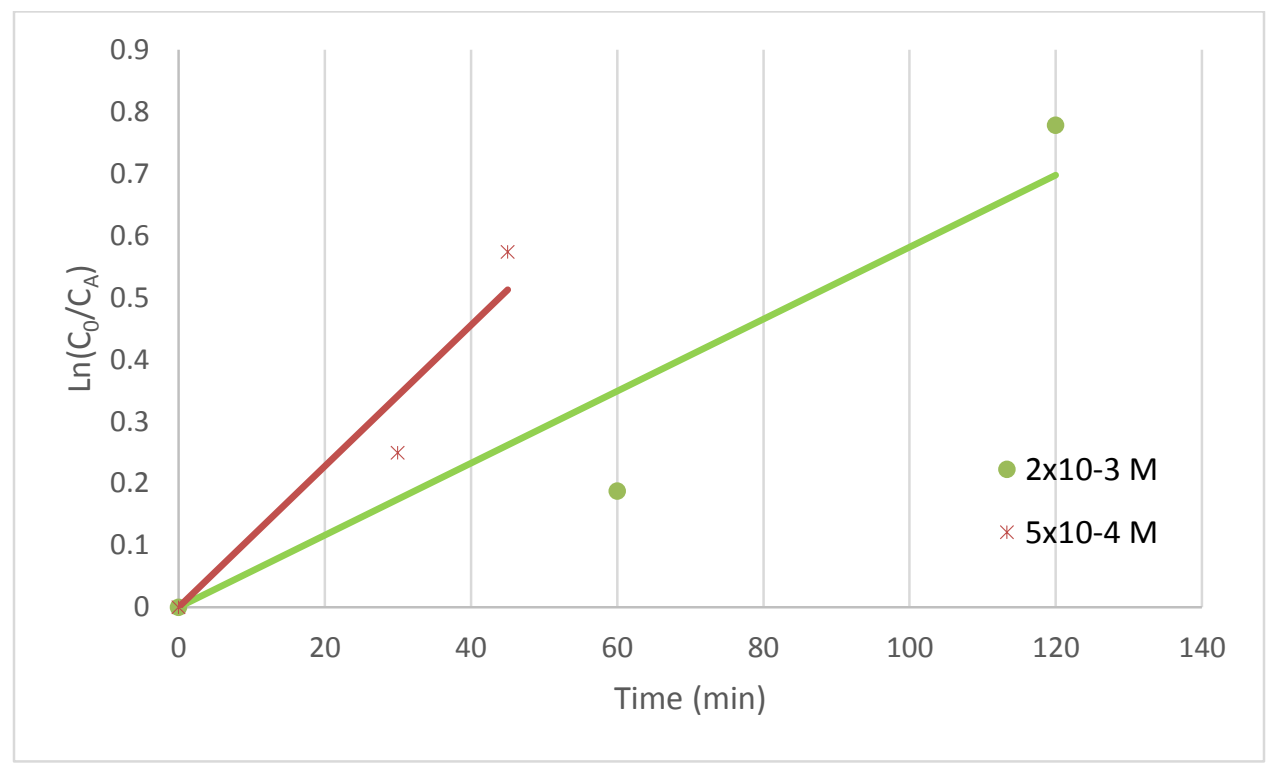

Figure 3-6 Time variation of MO concentration after the immersion of nickel NMFs with different concentration of MO solution 


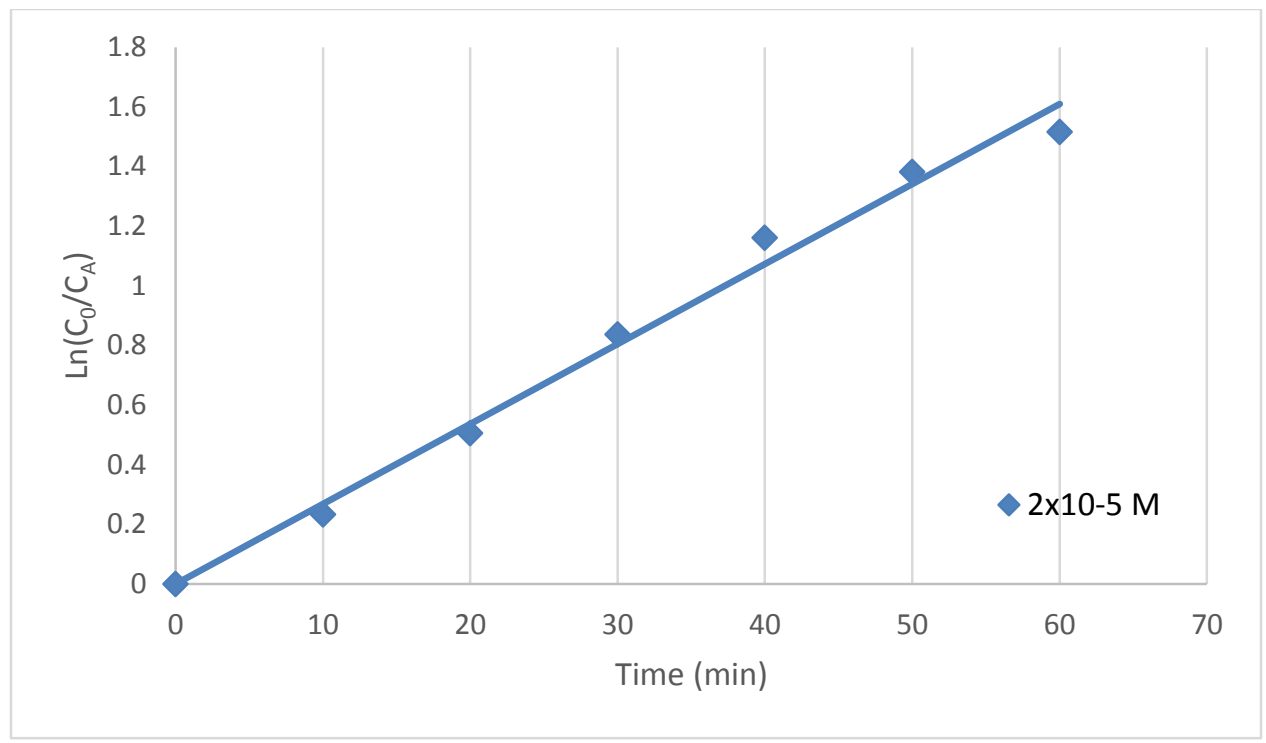

Figure 3-7 Time variation of MO concentration after the immersion of nickel NMFs with $2 \times 10^{-5} \mathrm{~mol} / \mathrm{L}$ MO solution

Figure 3-6 and 3-7 present the logarithmic curves of the kinetics for the degradation of MO solution. The pseudo first-order reaction model was supported by the data collected since the reaction constants, k's, were related to the initial concentration. Thus, a firstorder reaction model as followed is assumed and accepted for the kinetics behavior

$$
k t=\ln \left(\frac{C_{0}}{C_{A}}\right)
$$

The $k$ in the equation is the pseudo first-order rate constant. $C_{A}$ stands for the concentration at the testing time and $\mathrm{C}_{0}$ for the initial concentration. With the decrease of the concentration, the reaction rate increases obviously since the $k$ is related to the concentration of the agent involved. The values of $k$ and the $R^{2}$ are presented in the Table 1 below. 
Table 1 Kinetics constants for increased MO concentration

\begin{tabular}{|c|c|c|}
\hline Concentration $\left(\mathrm{mol} \mathrm{L}^{-1}\right)$ & Reaction Constant, $\mathrm{k}\left(\mathrm{min}^{-1}\right)$ & $\mathrm{R}^{2}$ \\
\hline $2.00 \mathrm{E}-05$ & 0.0268 & 0.9896 \\
\hline $5.00 \mathrm{E}-04$ & 0.0058 & 0.9017 \\
\hline $2.00 \mathrm{E}-03$ & 0.0114 & 0.9262 \\
\hline
\end{tabular}

Compared with the kinetics behavior reported by Masataka Hakamada et al.,(Hakamada, Hirashima et al. 2012) using the nanoporous nickel produced with dealloying process and the $\mathrm{MO}$ solution with the concentration of $2 \times 10^{-5} \mathrm{M}$, the decrease of concentration in our research is more rapid. The reaction rate they reported was at the level of $10^{-3}$, which was smaller than those we obtained in our research. Moreover, the concentration of the solution after 24 hours is even lower in our experiment. They reported that 24 hours were necessary to reduce the concentration ratio from 1 to 0.2 . However, the same ratio could be achieved within 1-2 hours in our research and the ratio would go down to 0.035 after 24 -hour-immersion. 


\section{CHAPTER 4}

\section{FUTURE WORK AND CONCLUSION}

\subsection{Future work}

Other than gold and nickel NMFs, synthesis methods of various nano-structured metals including silver, palladium, copper and iron have been developed for years. (Iravani and Zolfaghari 2013; Liu, Li et al. 2013; Xie, Ma et al. 2013; Snovski, Grinblat et al. 2014) Both silver and palladium nanoporous foams were successfully produced in our lab with microwave irradiation. The synthesis for nickel and platinum NMFs were developed in our lab as well, but some pressure issue came out during the microwave heating process. The pressure exceeded $250 \mathrm{psi}$, the safe pressure point for the microwave oven, after about a 3-minute-heating. Due to the problem, the heating would stop automatically and it would take at least 40 minutes to go back to the pressure release point, which greatly limited the producing rate of the synthesis. In order to solve this problem, we may try to vent during the microwave irradiation process. The difference was, through the synthesis process, nickel NMFs could be generated but platinum NMFs could not.

Future work for the research should include the development of synthesis for other nanoporous metal foams, such as copper, platinum, manganese and iron. The solution to the pressure problem happened during the synthesis process of nickel NMFs still need to figure out in order to improve the efficiency of the synthesis. Once the problem 
was solved, other applications such as the energy and hydrogen storage and ultra-highfield electromagnets will be able to be studied. Otherwise, applications of other nanoporous metals could be developed as well. For example, platinum NMFs can be applied as the glucose sensor the same as the nickel NMFs.(Park, Jeong et al. 2012) Moreover, different kind of reducing agents should be studied for the process of synthesis as the future work. As proved in our previous work, the surface properties are related to the reducing agents. It is study worthy to determine the better reducing agent for nanoporous metal foams.

Research about the lower detection limit of analyte is also included in future work, the detail structures of the NMFs surface need to be studied to analyze the reason why R6G cannot be detected in a lower concentration with the presence of gold NMFs. Future work may also include to improve the degradation rate of nickel NMFs to degrade $\mathrm{MO}$ in water.

\subsection{Conclusion}

Nanoporous metal foam is a three-dimensional structure with large porosities (greater than 0.4), and pore diameters between 1-100 $\mathrm{nm}$. It combines the outstanding properties of nanostructure such as low density, large surface area, high strength-toweight ratio and catalytic activity and properties of metals such as excellent electrical and thermal conductivity, good mechanical properties and stability.

We studied and developed new synthesis method to produce nanoporous metal foams with microwave irradiation heating. Besides the heating process, the reaction is 
basically a redox reaction from metal ions to molecules. Inorganic metal compound serves as the oxidant and hydrazine or sodium borohydride reacts as the reductant. Microwave irradiation process is applied to drive the redox reaction of metal compound forward. With EG or PVP and $\mathrm{NaCl}$ and ethanol presented as reaction-control reagent and dispersion media. Gold, silver, nickel and palladium have been researched for a long time and NMFs generated with these metal compounds obtained success in our lab by the microwave heating. Due to its properties of high efficiency and uniformly heating, relatively short heating time is required for the procedure and high surface area can be achieved.

Applications of the nanoporous metal foams produced are also presented, especially for gold and nickel NMFs. Gold NMFs is able to be applied to Surface Enhanced Raman Spectroscope as the substrate to enlarge the intensity of signal with relatively low concentration of $R 6 G$ solution. The lowest detection limit of $R 6 G$ in our research achieved $5.2 \times 10^{-7} \mathrm{M}$, compared to the fact that almost no detectable points existed in the concentration of $6 \times 10^{-5} \mathrm{M}$ without the nanoprous metal substrate. The prominent features at $612,773,1177,1307,1362$ and $1507 \mathrm{~cm}^{-1}$ were observed, which match with the previous work and theorem.

The application for green chemistry was presented as well. The nickel NMFs we produced can be used for the degradation of $\mathrm{MO}$ solution since it serves as an ideal catalyst for destruction of dye pollution in water with a low temperature and non-toxic process. MO solution with different concentrations were applied to study the catalytic ability if the nickel NMFs. The absorbance of the solution was observed to decrease significantly after immersed with $0.025 \mathrm{~g}$ nickel NMFs for 10 hours at room temperature 
and dark environment, which is a relatively rapid process compared with the previous work. The data analysis for the reaction kinetics provides that the reactions fit well as a pseudo first-order reaction model. It is worthy to note that in the case of degradation of $2 \times 10^{-5} \mathrm{M} M O$ solution, the degradation reaction rate is much higher and the concentration would decrease to $20 \%$ within 1 hour. The research outcome is beneficial for the removal of azo dyes in water treatment for industrial pollution. 


\section{Reference}

Albrecht, M. G. and J. A. Creighton (1977). "Anomalously intense Raman spectra of pyridine at a silver electrode." Journal of the American Chemical Society 99(15): 5215-5217.

Alyamani, A. and O. Lemine "FE-SEM Characterization of Some Nanomaterial."

Chan, S., S. Kwon, et al. (2003). "Surface-Enhanced Raman Scattering of Small Molecules from SilverCoated Silicon Nanopores." Advanced Materials 15(19): 1595-1598.

Dar, N., W. Wen-Jing, et al. (2011). High Surface-Enhanced Raman Scattering (SERS) sensitivity of R6G by fabrication of silver nanoparticles over GaN nanowires. Nanotechnology (IEEE-NANO), 2011 11th IEEE Conference on, IEEE.

E.Karthikeyan, S. R. a. (2011). "Microwave Synthesis - A Potential Tool for Green Chemistry." International Journal of ChemTech Research 3: 466-470.

Fleischmann, M., P. J. Hendra, et al. (1974). "Raman spectra of pyridine adsorbed at a silver electrode." Chemical Physics Letters 26(2): 163-166.

G Q Lu, X. S. Z. (2005). "Nanoporous Materials - An Overview." Nanoporous Materials: Science and Engineering: 12.

Gates, B. C. (2013). "Supported gold catalysts: new properties offered by nanometer and sub-nanometer structures." Chem Commun (Camb) 49(72): 7876-7877.

Hafner, B. (2007). "Scanning Electron Microscopy Primer." Characterization Facility, University of Minnesota-Twin Cities.

Hakamada, M., F. Hirashima, et al. (2012). "Catalytic decoloration of methyl orange solution by nanoporous metals." Catalysis Science \& Technology 2(9): 1814.

Han, K. K., Y. Zhou, et al. (2013). "One-pot synthesis of foam-like magnesia and its performance in CO2 adsorption." Microporous and Mesoporous Materials 169: 112-119.

He, X. N., Y. Gao, et al. (2012). "Surface-enhanced Raman spectroscopy using gold-coated horizontally aligned carbon nanotubes." Nanotechnology 23(20): 205702. 
Hu, B., S. B. Wang, et al. (2008). "Microwave-assisted rapid facile "green" synthesis of uniform silver nanoparticles: Self-assembly into multilayered films and their optical properties." Journal of Physical Chemistry C 112(30): 11169-11174.

Iravani, S. and B. Zolfaghari (2013). "Green Synthesis of Silver Nanoparticles Using Pinus eldarica Bark Extract." BioMed Research International 2013: 1-5.

Jeanmaire, D. L. and R. P. Van Duyne (1977). "Surface raman spectroelectrochemistry: Part I. Heterocyclic, aromatic, and aliphatic amines adsorbed on the anodized silver electrode." Journal of Electroanalytical Chemistry and Interfacial Electrochemistry 84(1): 1-20.

Kassu, A., P. Robinson, et al. (2010). Gold/silver coated nanoporous ceramic membranes: a new substrate for SERS studies.

Kim, T. K., K. J. Lee, et al. (2013). "Nanoporous Metal Oxides with Tunable and Nanocrystalline Frameworks via Conversion of Metal-Organic Frameworks." Journal of the American Chemical Society 135(24): 8940-8946.

Kneipp, K., M. Moskovits, et al. (2007). "Surface-enhanced Raman scattering." Physics Today 60(11): 40.

Kudelski, A. (2005). "Raman studies of rhodamine $6 G$ and crystal violet sub-monolayers on electrochemically roughened silver substrates: Do dye molecules adsorb preferentially on highly SERSactive sites?" Chemical Physics Letters 414(4-6): 271-275.

Kudelski, A. (2005). "Raman studies of rhodamine $6 G$ and crystal violet sub-monolayers on electrochemically roughened silver substrates: Do dye molecules adsorb preferentially on highly SERSactive sites?" Chemical Physics Letters 414(4): 271-275.

Kumar, R., G. Kumar, et al. (2013). "ZnO nano-mushrooms for photocatalytic degradation of methyl orange." Materials Letters 97: 100-103.

Li, J. F., Y. F. Huang, et al. (2010). "Shell-isolated nanoparticle-enhanced Raman spectroscopy." Nature 464(7287): 392-395.

Liu, C., W. Li, et al. (2013). Synthesis and size control of nano/submicron copper particles by feeding strategies. Nano/Micro Engineered and Molecular Systems (NEMS), 2013 8th IEEE International Conference on. 
Liu, Y.-C., C.-C. Yu, et al. (2006). "Low concentration rhodamine 6 G observed by surface-enhanced Raman scattering on optimally electrochemically roughened silver substrates." Journal of Materials Chemistry 16(35): 3546-3551.

Lu, W., X. Qin, et al. (2013). "Ni foam: a novel three-dimensional porous sensing platform for sensitive and selective nonenzymatic glucose detection." Analyst 138(2): 417-420.

Nadagouda, M. N., T. F. Speth, et al. (2011). "Microwave-Assisted Green Synthesis of Silver Nanostructures." Accounts of Chemical Research 44(7): 469-478.

Naya, M., T. Tani, et al. (2008). Nanophotonics bio-sensor using gold nanostructure.

$\mathrm{Ni}, \mathrm{W} ., \mathrm{H}$. B. Wu, et al. (2012). "One-Pot Synthesis of Ultra-Light Nickel Nanofoams Composed of Nanowires and Their Transformation into Various Functional Nanofoams." Small 8(22): 3432-3437.

Park, S., R. A. Jeong, et al. (2012). "Nonenzymatic continuous glucose monitoring in human whole blood using electrified nanoporous Pt." Biosensors and Bioelectronics 31(1): 284-291.

Qian, L. H., X. Q. Yan, et al. (2007). "Surface enhanced Raman scattering of nanoporous gold: Smaller pore sizes stronger enhancements." Applied Physics Letters 90(15): 153120.

Saini, G. S. S., S. Kaur, et al. (2005). "Spectroscopic studies of rhodamine 6 G dispersed in polymethylcyanoacrylate." Spectrochimica Acta Part A: Molecular and Biomolecular Spectroscopy 61(4): 653-658.

Smith, K. and C. Oatley (1955). "The scanning electron microscope and its fields of application." British Journal of Applied Physics 6: 391-399.

Snovski, R., J. Grinblat, et al. (2014). "Synthesis and characterization of iron, iron oxide and iron carbide nanostructures." Journal of Magnetism and Magnetic Materials 349: 35-44.

Sun, Y. (2002). "Shape-Controlled Synthesis of Gold and Silver Nanoparticles." Science 298(5601): 21762179.

Tappan, B. C., S. A. Steiner, 3rd, et al. (2010). "Nanoporous metal foams." Angew Chem Int Ed Engl 49(27): 4544-4565. 
Thirupathi, R. and E. N. Prabhakaran (2011). "Self-assembled microtubes and rhodamine 6G functionalized Raman-active gold microrods from 1-hydroxybenzotriazole." Journal of Chemical Sciences 123(3): 247-254.

Vernon-Parry, K. (2000). "Scanning electron microscopy: an introduction." III-Vs Review 13(4): 40-44.

Vo-Dinh, T. (1998). "Surface-enhanced Raman spectroscopy using metallic nanostructures." TrAC Trends in Analytical Chemistry 17(8-9): 557-582.

Wang, Y., Y.-F. Shi, et al. (2012). "Hydrazine reduction of metal ions to porous submicro-structures of Ag, $\mathrm{Pd}, \mathrm{Cu}, \mathrm{Ni}$, and Bi." Journal of Solid State Chemistry 191: 19-26.

Wiley, B., T. Herricks, et al. (2004). "Polyol Synthesis of Silver Nanoparticles: Use of Chloride and Oxygen to Promote the Formation of Single-Crystal, Truncated Cubes and Tetrahedrons." Nano Letters 4(9): 1733-1739.

Xie, T., Y. Ma, et al. (2013). "Controlled synthesis of snowflake-like self-assemblies palladium nanostructures under microwave irradiation." Materials Research Bulletin 48(8): 2850-2854.

Zhang, J., Y. Chen, et al. (2012). "Raman spectrum of carbon nanotubes coated by Au nano particles film." Zhongguo Jiguang/Chinese Journal of Lasers 39(11): 1115001. 